\title{
VISCOSITY METHODS FOR LARGE DEVIATIONS ESTIMATES OF MULTISCALE STOCHASTIC PROCESSES
}

\author{
DARIA GHILLI
}

\begin{abstract}
We study singular perturbation problems for second order HJB equations in an unbounded setting. The main applications are large deviations estimates for the short maturity asymptotics of stochastic systems affected by a stochastic volatility, where the volatility is modelled by a process evolving at a faster time scale and satisfying some condition implying ergodicity.
\end{abstract}

\section{INTRODUCTION}

We study the asymptotic behaviour as $\varepsilon, \delta \rightarrow 0$ of stochastic systems in the form

$$
\begin{cases}d X_{t}=\varepsilon \phi\left(X_{t}, Y_{t}\right) d t+\sqrt{2 \varepsilon} \sigma\left(X_{t}, Y_{t}\right) d W_{t} & X_{0}=x \in \mathbb{R}^{n}, \\ d Y_{t}=\frac{\varepsilon}{\delta} b\left(Y_{t}\right) d t+\sqrt{\frac{2 \varepsilon}{\delta}} \tau\left(Y_{t}\right) d W_{t} & Y_{0}=y \in \mathbb{R}^{m},\end{cases}
$$

where $\varepsilon, \delta>0, W_{t}$ is a standard $m$-dimensional Brownian motion and the matrix $\tau$ is non-degenerate. This is a model of system where the variables $Y_{t}$ evolve at a much faster time scale $s=\frac{t}{\delta}$ than the other variables $X_{t}$. The parameter $\varepsilon$ has been added to study the small time behaviour of the system, so time has been rescaled in (1.1) as $t \mapsto \varepsilon t$. Since we expect different limit behaviours depending on the rate $\frac{\varepsilon}{\delta}$, we choose $\delta=\varepsilon^{\alpha}$ with $\alpha \geq 2$. Motivated by the applications to large deviations that we want to give, we study the limit as $\varepsilon \rightarrow 0$ of the following logarithmic functional of the trajectories of (1.1)

$$
v^{\varepsilon}(t, x, y):=\varepsilon \log E\left[e^{h\left(X_{t}\right) / \varepsilon} \mid(X ., Y .) \text { satisfy }(1.1)\right],
$$

where $h$ is a bounded continuous function and we characterize $v^{\varepsilon}$ as the solution of the Cauchy problem with initial data $v^{\varepsilon}(0, x, y)=h(x)$ for a fully nonlinear parabolic equation in $n+m$ variables.

Our first interest is to characterize the limit $v$ of the functions $v^{\varepsilon}$ as the solution of the Cauchy problem for a first order Hamilton-Jacobi equation in $n$ space dimensions

$$
\left.v_{t}-\bar{H}(x, D v)=0 \quad \text { in }\right] 0, T\left[\times \mathbb{R}^{n}, \quad v(0, x)=h(x),\right.
$$

for a suitable effective Hamiltonian $\bar{H}$. This is a singular perturbation problem for the system (1.1) which we treat by viscosity solutions methods for HJ equations.

The primary motivation for the study of systems of the form (1.1) comes from mathematical models of financial markets with stochastic volatility, where the asset prices are affected by correlated economic factors and the volatility $\sigma$ is modelled as a function of an

Date: 16.05.2016.

1991 Mathematics Subject Classification. 35XX.

Key words and phrases. viscosity solutions; Hamilton-Jacobi-Bellman equations; homogenization and singular perturbations; large deviations; stochastic volatility models.

This work was partially supported by the ERC advanced grant 668998 (OCLOC) under the EU's H2020 research programme. 
Ito process driven by another Brownian motion. This approach seems to have the advantage of taking into account the so called smile effect (caused by the discrepancy between the predicted and market traded option prices) and reproducing more realistic-with fatter and asymmetric tails-returns distributions. Recently an extension to this approach was proposed by Fouque, Papanicolaou, and Sircar in [27], with the aim to model the bursty behaviour of volatility observed in financial markets (that is, the empirically observed tendence of fluctuating to high level and then to low level for small time periods). These phenomena are also related to another feature of volatility, which is mean reversion. The mathematical framework of multiple time scales systems and singular perturbations takes into account both these phenomena.

Following this approach, we model the log-prices of the asset with the slow variable $X_{t}$ and the volatility $\sigma$ with a process $Y_{t}$ evolving on a faster time scale $s=\frac{t}{\delta}$ and which is ergodic, that is, it has a unique invariant long-run distribution and asymptotically decorrelates. Here $\delta$ is the fast mean reversion time and it is smaller than the order of maturity $\varepsilon$. The asymptotic analysis as $\varepsilon, \delta \rightarrow 0$ gives a simple pricing and hedging theory taking into account the effect of a changing volatility and then providing a correction to the classical Black-Scholes formulas.

The existing techniques to treat singular perturbations problems as (1.2) have been developed so far mainly under assumptions implying some kind of compactness of the fast variable. We refer mainly to the methods of [4], stemming from Evans' perturbed test function method for homogenization [23] and its extensions to singular perturbations $[1,2,3]$. A standard hypothesis is for example the periodicity of the coefficients of the stochastic system with respect $Y_{t}$, which in particular implies the periodicity in $y$ of the solutions $v^{\varepsilon}$ (see e.g. [7] and the end of the introduction for more details).

Fundamental aim of this paper is to consider unbounded fast processes by replacing the compactness with some condition implying ergodicity. A quite natural condition is the following

$$
b(y) \cdot y \leq-B|y|^{2}, \quad \text { if }|y|>R, \quad \text { for some } B>0, R>0
$$

which is reminiscent of other similar conditions about recurrence of diffusion processes in the whole space (see for example [6], [39], [40], [41]). The main example is the OrnsteinUhlenbeck process. The interest in the analysis of such kind of systems is in part related to the financial applications we have in mind; in particular, the standard compactness/periodicity assumptions appear as too restrictive in order to model volatility in financial markets, see the empirical data and the discussion presented in [27] and the references therein.

We identify and study two different regimes depending on how fast the volatility oscillates relative to the horizon length, namely the ultra-fast mean reverting regime $\delta=\varepsilon^{\alpha}$ (supercritical case), with $\alpha>2$ and fast mean reverting regime $\delta=\varepsilon^{2}$ (critical case). The subcritical case $\alpha<2$ is not analysed in the present paper, since the ergodic problem arising in this case can not be solved (see Remark 2.5 for more details). This seems to be linked to the fact that, in the slow mean reversion regime $(\alpha<2)$, the long-time behaviour of the $Y_{t}$ process does not come into play.

The first step is the identification of the effective Hamiltonians in both cases through the resolution of two different ergodic problems. For $\alpha>2$ the ergodic problem is finding, for any $(\bar{x}, \bar{p}) \in \mathbb{R}^{n} \times \mathbb{R}^{n}$, a couple $\lambda \in \mathbb{R}$ and $w$-viscosity solution of the following uniformly elliptic linear equation

$$
\lambda-\operatorname{tr}\left(\tau \tau^{T}(y) D^{2} w(y)\right)-b(y) \cdot D w(y)-\left|\sigma(\bar{x}, y)^{T} \bar{p}\right|^{2}=0 .
$$


Note that $\lambda=\bar{H}(\bar{x}, \bar{p})$ is the effective Hamiltonian and we call $w$ the corrector by analogy with the theory of homogenization. In order to prove the existence of the effective Hamiltonian and of the corrector we approximate the ergodic problem by the so-called approximate $\delta$-ergodic problem, namely

$$
\delta w_{\delta}(y)-\operatorname{tr}\left(\tau \tau^{T}(y) D^{2} w_{\delta}(y)\right)-b(y) \cdot D w_{\delta}(y)-\left|\sigma(\bar{x}, y)^{T} \bar{p}\right|^{2}=0 .
$$

The main result which allows us to conclude the existence is a $\delta$-uniform local Lipschitz bound for $w_{\delta}$ (see Lemma 4.3). For the uniqueness of the effective Hamiltonian, we rely on the ergodicity of the process $Y_{t}$ (encoded by the assumption (1.3)) and on the results of Bardi, Cesaroni, Manca [6], where the effective Hamiltonian is uniquely determined by the explicit formula

$$
H(\bar{x}, \bar{p})=\int_{\mathbb{R}^{m}}\left|\sigma(\bar{x}, y)^{T} \bar{p}\right|^{2} d \mu(y),
$$

where $\mu$ is the invariant probability measure on $\mathbb{R}^{m}$ of the stochastic process

$$
d Y_{t}=b\left(Y_{t}\right) d t+\sqrt{2} \tau\left(Y_{t}\right) d W_{t} .
$$

In addition, we prove the existence of the corrector, which is not investigated in [6].

For $\alpha=2$ the ergodic problem is finding, for any $(\bar{x}, \bar{p}) \in \mathbb{R}^{n} \times \mathbb{R}^{n}$, a couple $\lambda \in \mathbb{R}$ and $w$-viscosity solution of the following uniformly elliptic nonlinear equation

$\lambda-\operatorname{tr}\left(\tau \tau^{T}(y) D^{2} w(y)\right)-\left|\tau(y)^{T} D w(y)\right|^{2}-\left(b(y)+\tau(y) \sigma(\bar{x}, y)^{T} \bar{p}\right) \cdot D w(y)-\left|\sigma(\bar{x}, y)^{T} \bar{p}\right|^{2}=0$.

Differently from (1.4), equation (1.5) is nonlinear with quadratic growth in the gradient. We remark that the correlation term $\tau(y) \sigma(\bar{x}, y)^{T} \bar{p} \cdot D w(y)$ arises only in the fast mean reverting regime, whereas it does not play any role in the ultra-fast mean reverting case (see Remark 4.1). Note that in the cell problems we use the notation $D$ without subscripts for the gradient of the solution with respect to the $y$ variable.

For the existence of the effective Hamiltonian and the corrector, we proceed analogously to the supercritical case, in particular we rely on an analogous $\delta$-uniform local Lipschitz bound for the solution of the approximate $\delta$-ergodic problem (see Lemma 3.3). We prove the uniqueness of the effective Hamiltonian relying on the results by Ichihara [30], where ergodic problems for Bellman equations (in the case of a nonlinear quadratic term) are solved. For a representation formula we refer to [33], where $\bar{H}$ is written as the convex conjugate of a suitable operator over a space of measures.

Then, the crucial step is the convergence of the functions $v^{\varepsilon}$ to the solution of the limit problem (1.2). The difficulties stem primarily from the unboundedness of the fast variable, and the standard methods relying on the compactness/periodicity of the fast variable have to be modified. Our strategy rely on the following assumption on the fast process

$$
b(y)=b-y, \quad \tau(y)=\tau \quad \text { for }|y| \geq R_{1}
$$

for some $R_{1}>0$, where $b \in \mathbb{R}^{m}$ is a vector, and $\tau$ is bounded and uniformly non-degenerate. In particular, note that (1.6) is stronger as the usual condition implying ergodicity as (1.3) and it is satisfied e.g. by the Ornstein-Uhlenbeck process.

Our techniques are based on the perturbed test function method of [23], [4], with some relevant adaptations to the unbounded setting. We mainly rely on the ergodicity of the fast process through the use of a Liapounov function (see Section 2, subsection 2.3) into the perturbed test function. Further difficulties in the proof of the convergence come from the nonlinearity of the equation satisfied by the $v^{\varepsilon}$. A fundamental result on which we rely in the proof of the convergence is given in Proposition 5.1, that is, a global $\delta$-uniform Lipschitz bound for the solution of the approximate $\delta$-ergodic problems. 
A significant part of the paper is devoted to the proof of Proposition 5.1, which could be considered our main mathematical contribution. Indeed, not only it is a crucial step to prove the convergence, as already mentioned, but also it is a non standard result, at least to our knowledge, for the type of equations we consider, namely uniformly elliptic equations either with linear Hamiltonians in the gradient (supercritical case), or with superlinear quadratic Hamiltonians (critical case). The proof is in some part inspired by a method due to Ishii and Lions [32] (see also [20],[12] and the references therein), which essentially allows to take profit of the uniform ellipticity of the equation to control the Hamiltonian terms. However, we remark that usually the Ishii-Lions method allows to achieve bounds which depend on the $L^{\infty}$-norm of the solution (at least if we do not assume any periodicity), whereas our result is a global estimate in all the space independent of such norm. The fundamental hypothesis which enables us to achieve our result is the Ornstein-Uhlenbeck nature of the fast process at infinity encoded by assumption (1.6).

We recall some results in the literature related to gradient bounds for similar kinds of equations. Gradient bounds for superlinear-type Hamiltonians can be found in Lions [35] and Barles [9], see also Lions and Souganidis [37], Barles and Souganidis [14] and more, recently, Cardaliaguet and Silvestre [19] for nonlinear degenerate parabolic equations. However, we remark that in the previous works the bounds depend usually on the $L^{\infty}$ norm of the solution. In [14] some results independent of the $L^{\infty}$-norm of the solutions are established but in periodic environments. We recall also the result of [17] by CapuzzoDolcetta, Leoni, Porretta for coercive superlinear Hamiltonians, where a uniform gradient bound is proved, but in some Hölder norm and only in bounded domains. Recently, uniform Lipschitz bounds on the torus for analogous equations as ours (and more general) have been established by Ley and Duc Nguyen in [34].

Our second aim is to derive a large deviation principle for the process $X_{t}^{\varepsilon}$ as a first application of our convergence result. More specifically, following the approach of [25] and [7], we prove that the measures associated to the process $X_{t}$ in (1.1) satisfy such a principle with good rate function

$$
I\left(x ; x_{0}, t\right):=\inf \left[\int_{0}^{t} \bar{L}(\xi(s), \dot{\xi}(s)) d s \mid \xi \in A C(0, t), \xi(0)=x_{0}, \xi(t)=x\right],
$$

where $\bar{L}$ is the effective Lagrangian associated to $\bar{H}$ via convex duality. In particular we get that

$$
P\left(X_{t}^{\varepsilon} \in B\right)=e^{-\inf _{x \in B} \frac{I\left(x ; x_{0}, t\right)}{\varepsilon}+o\left(\frac{1}{\varepsilon}\right)}, \text { as } \varepsilon \rightarrow 0
$$

for any open set $B \subseteq \mathbb{R}^{n}$. Then, we apply this result to find estimates of option prices near maturity and an asymptotic formula for the implied volatility. We refer to Section 8 for further details.

An important reference to us is the paper [7], where the author together with M. Bardi and A. Cesaroni studied small time behaviour for the system defined above under the main assumption of periodicity on the coefficients. We remark that in [7] the resolution of the ergodic problems and the convergence rely deeply on the assumption of periodicity, which allows to take profit of more standard techniques. On the contrary and as already observed, the unboundedness of the fast process assumed in the present paper entails much more difficulties in the proofs, both in the resolution of the ergodic problems (that, is in the local gradient estimates and in the existence and uniqueness of $\bar{H}$ ) and mainly in the convergence result. However, note that in [7], a rather complete analysis is carried out, considering also the case $1<\alpha<2$, and several representation formulas for the effective Hamiltonian are given. Finally, similar financial applications to the ones above mentioned 
are also given in [7] (see Theorem 7.1, Corollaries 8.1 and 8.2). However, we underline that these financial applications take on particular relevance in the present paper due to the unbounded setting, which provides a more realistic model for volatility as appearing in financial markets.

We finally recall the paper [25], where Feng, Fouque, and Kumar study analogous problems for system of the form we consider, only for $\alpha=2$ and $\alpha=4$, in the one-dimensional case $n=m=1$, assuming that $Y_{t}$ is the Ornstein-Uhlenbeck process and the coefficients in the equation for $X_{t}$ do not depend on $X_{t}$. Their methods are based on the approach to large deviations developed in [26]. Comparing to [25], we remark that we consider more general fast processes satisfying (1.6), we treat vector-valued processes with $\phi$ and $\sigma$ depending on $X_{t}$ in a rather general way and we study all the range $\alpha \geq 2$. Also, our methods are different, mostly from the theory of viscosity solutions for fully nonlinear PDEs and from the theory of homogenization and singular perturbations for such equations.

Organization of the paper. In Section 2 we give the assumptions on the stochastic volatility model and we recall some preliminaries. In Sections 3 and 4 we analyse the ergodic problem and the properties of the effective Hamiltonian in the critical $(\alpha=2)$ and supercritical case $(\alpha>2)$, respectively. Section 5 is devoted to the proof of the Lipschitz bounds for the solution of the ergodic problems for each regimes. In Section 6 we prove the comparison principle for the limit equation (1.2) and in Section 7 we prove the convergence result for each regime of the functions $v^{\varepsilon}$ to the unique viscosity solution of the limit problem (1.2) with $\bar{H}$ identified in the previous sections. Finally in Section 8 we derive a large deviation principle for the stochastic system and we give some financial applications to estimate option prices and asymptotic volatility.

\section{Assumptions AND PRELIMINARIES}

2.1. The stochastic volatility model. We consider fast mean-reverting processes of the following type

$$
\begin{cases}d X_{t}=\phi\left(X_{t}, Y_{t}\right) d t+\sqrt{2} \sigma\left(X_{t}, Y_{t}\right) d W_{t}, & X_{0}=x \in \mathbb{R}^{n} \\ d Y_{t}=\frac{1}{\varepsilon^{\alpha}} b\left(Y_{t}\right) d t+\sqrt{\frac{2}{\varepsilon^{\alpha}}} \tau\left(Y_{t}\right) d W_{t}, & Y_{0}=y \in \mathbb{R}^{m},\end{cases}
$$

where $\varepsilon>0, \alpha \geq 2, \phi: \mathbb{R}^{n} \times \mathbb{R}^{m} \rightarrow \mathbb{R}^{n}, \sigma: \mathbb{R}^{n} \times \mathbb{R}^{m} \rightarrow \mathbf{M}^{n, m}$ are bounded functions, Lipschitz continuous in $(x, y), b: \mathbb{R}^{m} \rightarrow \mathbb{R}^{m}$ is Lipschitz continuous, $\tau: \mathbb{R}^{m} \rightarrow \mathbf{M}^{m, m}$ is bounded, Lipschitz continuous and uniformly non degenerate, i.e. satisfies for some $\theta>0$

$$
\xi^{T} \tau(y) \tau(y)^{T} \xi=\left|\tau^{T}(y) \xi\right|^{2}>\theta|\xi|^{2} \quad \text { for every } y \in \mathbb{R}, \xi \in \mathbb{R}^{m} .
$$

These assumptions will hold throughout the paper.

In order to study small time behaviour of the system (2.1), we rescale time $t \rightarrow \varepsilon t$, for $0<\varepsilon \ll 1$, so that the typical maturity will be of order $\varepsilon$. Denoting the rescaled process by $X_{t}^{\varepsilon}, Y_{t}^{\varepsilon}$, we get

$$
\left\{\begin{array}{lll}
d X_{t}^{\varepsilon}=\varepsilon \phi\left(X_{t}^{\varepsilon}, Y_{t}^{\varepsilon}\right) d t+\sqrt{2 \varepsilon} \sigma\left(X_{t}^{\varepsilon}, Y_{t}^{\varepsilon}\right) d W_{t}, & & X_{0}^{\varepsilon}=x \in \mathbb{R}^{n} \\
d Y_{t}^{\varepsilon}=\varepsilon^{1-\alpha} b\left(Y_{t}^{\varepsilon}\right) d t+\sqrt{2 \varepsilon^{1-\alpha}} \tau\left(Y_{t}^{\varepsilon}\right) d W_{t}, & Y_{0}^{\varepsilon}=y \in \mathbb{R}^{m}
\end{array}\right.
$$

The basic assumption on the drift $b$ is the following.

(E) There exist $B>0$ and $R$ such that

$$
b(y) \cdot y \leq-B|y|^{2}, \quad \text { if }|y|>R ;
$$

Remark 2.1. Assumption (E) implies the ergodicity of the fast process and, in particular, the existence of a Liapounov function (see subsection 2.3). This assumption is fundamental 
for the resolution of the ergodic problems and the identification of the effective Hamiltonians and it will be used in Sections 2,3,4. On the other hand, we remark that (E) is not enough to prove the convergence result, hence it will be be strengthened in Sections 5,7 by the following assumption (U).

The main assumptions on the drift $b$ and the volatility $\sigma$ are the following.

(U) There exist $b \in \mathbb{R}^{m}, \tau \in \mathbf{M}^{m, m}$ and $R_{1}$ such that

$$
b(y)=b-y, \quad \tau(y)=\tau \quad \text { if }|y|>R_{1} .
$$

(S1) Let $\alpha>2$. Then, for each $x \in \mathbb{R}^{n}$, there exists $\nu>0$ such that

$$
\left|\sigma(x, y)^{T} \xi\right|^{2} \geq \nu|\xi|^{2}, \quad \text { for all } y \in \mathbb{R}^{n}, \xi \in \mathbb{R}^{m} \text {. }
$$

(S2) Let $\alpha=2$. Then, for all $x \in \mathbb{R}^{n}$, there exists $g: \mathbb{R}^{m} \times \mathbb{R}^{m} \rightarrow \mathbb{R}^{+}$such that

$$
\|\sigma(x, y)-\sigma(x, z)\|_{\infty} \leq g(y, z)|y-z| \text { for all } y, z \in \mathbb{R}^{m},
$$

and $\forall \eta>0$, there exists $R_{\eta}>0$ such that $g(y, z) \leq \eta$ as $|z|,|y| \geq R_{\eta}$.

Remark 2.2. As already remarked, (U) is stronger than (E) (and therefore implies the ergodicity of the fast process) and it will be fundamental in Sections 5, 7 in order to prove the global Lipschitz bound for the corrector (Proposition 5.2) and the convergence result. For example, (U) is satisfied by Ornstein-Uhlenbeck type processes, i.e. processes $Y_{t}$ as in (2.3) such that

$$
b(y)=b-y, \quad \tau(y)=\tau \quad \text { for any } y \in \mathbb{R}^{m},
$$

for some $b \in \mathbb{R}^{m}$ and $\tau \in \mathbf{M}^{m, m}$ non-degenerate. The Ornstein-Uhlenbeck process is a classical example of a Gaussian process that admits a stationary probability distribution and in particular is a mean-reverting process, namely there is a long-term value towards the process "tends to revert". However, we remark that (U) is more general than (2.4) since it is required only outside some compact which can be arbitrarily big.

Remark 2.3. Assumption (S2) says, roughly speaking, that the Lipschitz constant of $\sigma(x, \cdot)$, considered as a function on $\mathbb{R}^{m}$ for $x \in \mathbb{R}^{n}$ fixed, vanishes at infinity. At least to our point of view, (S2) seems not restrictive in the context of financial models, since it influences the behaviour of $\sigma$ only at infinity, which in general is not "seen" in the financial applications we are interested in. Examples of sufficient conditions for (S2) are

$$
\begin{aligned}
& \lim _{|y| \rightarrow+\infty} g(y, z)=0 \quad \text { uniformly in } z, \\
& \lim _{|z| \rightarrow+\infty} g(y, z)=0 \quad \text { uniformly in } y .
\end{aligned}
$$

For example, the above conditions are satisfied by $\sigma(x, y)=\frac{1}{\left(1+|y|^{2}\right)^{\alpha}}$, for $\alpha>0$. Then we have (S2) with $g(y, z)=\frac{C}{1+|y|+|z|}$. Without loss of generality we suppose $n=1$ and $z \geq y \geq 0$. Then

$$
\sigma(y)-\sigma(z)=\frac{1}{\left(1+y^{2}\right)^{\alpha}}\left(1-\left(1+\frac{y^{2}-z^{2}}{1+z^{2}}\right)^{\alpha}\right) .
$$

From the inequality $1-(1+x)^{\alpha} \leq-x$ for $-1 \leq x \leq 0$, we get

$$
\sigma(y)-\sigma(z) \leq \frac{1}{\left(1+y^{2}\right)^{\alpha}} \frac{(z-y)(z+y)}{1+z^{2}} \leq \frac{2 z}{1+z^{2}}(y-z) .
$$

Since we assumed $z \geq y \geq 0$, we can find a constant $C$ independent of $y, z$ such that $\frac{2 z}{1+z^{2}} \leq \frac{C}{1+z+y}$, concluding the proof. 
Assumption (S2) is used to prove the Lipschitz bound for the corrector in the critical case (Proposition 5.1). In particular, we need to assume (S2) to treat the correlation term $\tau(y) \sigma^{T}(\bar{x}, y) \bar{p} \cdot D w_{\delta}$, which appears in the ergodic problem for $\alpha=2$. On the contrary, for $\alpha>2$ the correlation term do not appear in the ergodic problem (see (4.3) in the following) and then we do not need assumption (S2).

Assumption (S1) of uniform non-degeneracy of the volatility is due to technical issues arising in the proof of the local gradient bound for the solution of the ergodic problem for $\alpha>2$. We refer in particular to the proof of Lemma 4.3.

Remark 2.4. Note that in the system (2.3) the processes $X_{t}$ and $Y_{t}$ are driven by the same Brownian motion $W_{t}$, allowing strong correlation. Also, note that the case of a system as in (2.3) with two different Brownian motions for $X_{t}$ and $Y_{t}$ respectively, can be written in the form of (2.3) by suitably redefining the coefficients, in particular, by including the correlation term inside the matrix of the diffusion of the fast process (see also [6], Section 6 for more details).

2.2. The logarithmic transformation method and the HJB equation. We consider the following functional

$$
v^{\varepsilon}(t, x, y):=\varepsilon \log E\left[e^{h\left(X_{t}\right) / \varepsilon} \mid(X ., Y .) \text { satisfy }(2.3)\right],
$$

where $h \in B C\left(\mathbb{R}^{n}\right)$ and $\left(X_{s}, Y_{s}\right)$ satisfies (2.3). Note that the logarithmic form of the functional in (2.5) is motivated by the applications to large deviations that we want to give.

A standard result is that $v^{\varepsilon}$ can be characterized as the unique continuous viscosity solution of the following parabolic problem. We refer to Da Lio and Ley in [21] for a proof.

Proposition 2.1. Let $\alpha \geq 2$ and define

$$
\begin{aligned}
H^{\varepsilon}(x, y, p, q, X, Y, Z) & :=\left|\sigma^{T} p\right|^{2}+b \cdot q+\operatorname{tr}\left(\tau \tau^{T} Y\right)+\varepsilon\left(\operatorname{tr}\left(\sigma \sigma^{T} X\right)+\phi \cdot p\right) \\
& +2 \varepsilon^{\frac{\alpha}{2}-1}\left(\tau \sigma^{T} p\right) \cdot q+2 \varepsilon^{\frac{1}{2}} \operatorname{tr}\left(\sigma \tau^{T} Z\right)+\varepsilon^{\alpha-2}\left|\tau^{T} q\right|^{2} .
\end{aligned}
$$

Then $v^{\varepsilon}$ is the unique bounded continuous viscosity solution of the Cauchy problem

$$
\begin{cases}\partial_{t} v^{\varepsilon}-H^{\varepsilon}\left(x, y, D_{x} v^{\varepsilon}, \frac{D_{y} v^{\varepsilon}}{\varepsilon^{\alpha-1}}, D_{x x}^{2} v^{\varepsilon}, \frac{D_{y y}^{2} v^{\varepsilon}}{\varepsilon^{\alpha-1}}, \frac{D_{x y}^{2} v^{\varepsilon}}{\varepsilon^{\frac{\alpha-1}{2}}}\right)=0 & \text { in }[0, T] \times \mathbb{R}^{n} \times \mathbb{R}^{m}, \\ v^{\varepsilon}(0, x, y)=h(x) & \text { in } \mathbb{R}^{n} \times \mathbb{R}^{m} .\end{cases}
$$

Remark 2.5. We treat the range $\alpha \geq 2$ and we do not deal with the case $\alpha<2$. Indeed, for $\alpha<2$, the ergodic problem is finding (and characterizing it uniquely) $\lambda \in \mathbb{R}$ and a $w$-viscosity solution of the following equation:

$$
\lambda-2\left(\tau(y) \sigma(\bar{x}, y)^{T} \bar{p}\right) \cdot D_{y} w(y)-\left|\tau(y)^{T} D_{y} w(y)\right|^{2}-\left|\sigma(\bar{x}, y)^{T} \bar{p}\right|^{2}=0
$$

which is not solvable in general. This seems to be linked to the fact that in the slow mean reversion regime $(\alpha<2)$, the long-time behaviour of the $Y_{t}$ process does not come into play. Indeed, the ergodicity of the fast process plays no role in $(2.7)$, since the cost $\left(\left|\sigma^{T} \bar{p}\right|^{2}\right)$ and the drift $2 \tau \sigma^{T} \bar{p}$ are both bounded and the drift $b$ has disappeared. On the contrary, in the case $\alpha \geq 2$, this role is played by the term $-b \cdot D w$ where $b$ satisfies assumption (E). Finally we recall that in [7] the case $\alpha<2$ is solved thanks to the periodicity assumption.

2.3. A Liapounov-like condition. In this section we prove the existence of a Liapounov function for the following operator

$$
\mathcal{G}_{\bar{x}, \bar{p}}(y, q, Y)=-\left(b(y)+2 \tau(y) \sigma^{T}(\bar{x}, y) \bar{p}\right) \cdot q-\left|\tau^{T}(y) q\right|^{2}-\operatorname{tr}\left(\tau \tau^{T}(y) Y\right),
$$


i.e. we prove that for each $(\bar{x}, \bar{p}) \in \mathbb{R}^{n} \times \mathbb{R}^{n}$ there exists a continuous function $\chi_{\bar{x}, \bar{p}}:=\chi$, such that $\chi(y) \rightarrow+\infty$ as $|y| \rightarrow+\infty$ and if $\mathcal{G}[\chi]:=\mathcal{G}_{\bar{x}, \bar{p}}\left(y, D \chi(y), D^{2} \chi(y)\right)$ then

$$
\mathcal{G}[\chi] \rightarrow+\infty \text { as }|y| \rightarrow+\infty \text { in the viscosity sense. }
$$

The existence of a Liapounov function is reminiscent of other similar conditions about ergodicity of diffusion processes in the whole space; see, for example [29],[36], [15], [16], [38].

Remark 2.6. We observe that

$$
\mathcal{G}_{\bar{x}, \bar{p}}(y, q, Y)=-\mathcal{L}_{\bar{x}, \bar{p}}(y, q, Y)-\left|\tau^{T}(y) q\right|^{2}
$$

where, for any $(\bar{x}, \bar{p}) \in \mathbb{R}^{n} \times \mathbb{R}^{n}, \mathcal{L}_{\bar{x}, \bar{p}}$ is the linear operator

$$
\mathcal{L}_{\bar{x}, \bar{p}}(y, q, Y)=\left(b(y)+2 \tau(y) \sigma^{T}(\bar{x}, y) \bar{p}\right) \cdot q+\operatorname{tr}\left(\tau \tau^{T}(y) Y\right),
$$

which is the infinitesimal generator of the stochastic process

$$
d Y_{t}=\left(b\left(Y_{t}\right)+2 \tau\left(Y_{t}\right) \sigma^{T}\left(\bar{x}, Y_{t}\right) \bar{p}\right) d t+\tau\left(Y_{t}\right) d W_{t} .
$$

Note that we consider the additional term $-\left|\tau^{T} q\right|^{2}$ in (2.9) and this is due to the logarithmic form of the value function $v_{\varepsilon}$ defined in (2.5), which is in turn motivated by the applications to large deviations we are interested in.

Now we prove the following lemma.

Lemma 2.2. Let $(E)$ hold. Then for any $(\bar{x}, \bar{p}) \in \mathbb{R}^{n} \times \mathbb{R}^{n}$ there exists a Liapounov-like function for the operator $\mathcal{G}_{\bar{x}, \bar{p}}$.

Proof. Note that a key role in the following proof is played by the behaviour of the drift $b$ at infinity, which is encoded by assumption (E).

We take

$$
\chi=a|y|^{2},
$$

and by (E) and the boundedness of $\tau$, we have for $|y| \geq R$

$$
-b(y) \cdot D \chi(y)-\left|\tau^{T}(y) D \chi(y)\right|^{2} \geq 2 a B|y|^{2}-4 a^{2} T|y|^{2}-2 a|b||y|,
$$

where $T>0$ depends on $\|\tau\|_{\infty}$. Then by taking

$$
a<\frac{B}{2 T},
$$

the other terms in $\mathcal{G}$ being negligible because of the boundedness of $\tau$ and $\sigma$, we finally get $(2.8)$.

Remark 2.7. We observe that condition (E) reminds classical conditions for ergodicity, see for example [6]. In particular we recall the so-called recurrence condition used by Pardoux and Veretennikov [39], [40], [41] namely

$$
b(y) \cdot y \rightarrow-\infty \quad \text { as }|y| \rightarrow+\infty .
$$

Note that (E) is stronger than (2.13). The main reason is that in our context we need to have some additional information on the rate of decay of $b \cdot y$, in particular we need it to be at least quadratic in order to compete with the quadratic growth (in the gradient term) of $\mathcal{G}$ (see also Remark 2.6). 


\section{The CRITICAL CASE: $\alpha=2$}

3.1. Key preliminary results. For any $(\bar{x}, \bar{p}) \in \mathbb{R}^{n} \times \mathbb{R}^{n}$, the ergodic problem is finding a constant $\lambda \in \mathbb{R}$ such that the following equation

$\lambda-\operatorname{tr}\left(\tau \tau^{T}(y) D^{2} w(y)\right)-\left|\tau^{T}(y) D w\right|^{2}-\left(b(y)+2\left(\tau(y) \sigma^{T}(\bar{x}, y) \bar{p}\right) \cdot D w(y)-\left|\sigma(\bar{x}, y)^{T} \bar{p}\right|^{2}=0\right.$.

has a viscosity solution $w$. This kind of ergodic problems have been studied by Ichihara [30] and Ichihara and Sheu [31]. We refer in particular to Theorem 2.4 of [30], which we recall in the following proposition.

Denote

$$
\Phi=\left\{w \in C^{2}\left(\mathbb{R}^{m}\right): \text { there exists } C<0 \text { such that } w(y) \leq C(1+|y|)\right\} .
$$

Proposition 3.1. Let assumption (E) hold. There exists a constant $\lambda^{*} \in \mathbb{R}$ such that (3.1) admits a classical solution $w \in C^{2}\left(\mathbb{R}^{m}\right)$ if and only if $\lambda \leq \lambda^{*}$. Moreover, if $(\lambda, w)$ is a solution of (3.1) and $w \in \Phi$, then $\lambda=\lambda^{*}$.

Remark 3.1. We remark that Theorem 2.4 is proved for Hamiltonians which are convex in the gradient variable, whereas in our case the Hamiltonian is concave. The two cases are equivalent, since if we have a solution $w$ of (3.1), then $-w$ is a solution of

$$
-\lambda-\operatorname{tr}\left(\tau \tau^{T}(y) D^{2} w(y)\right)+H(y, D w(y))=0,
$$

where

$$
H(y, q)=-b(y) \cdot q+\left|\tau(y)^{T} q\right|^{2}-2 \tau(y) \sigma(\bar{x}, y)^{T} \bar{p} \cdot q+|\sigma(\bar{x}, y) \bar{p}|^{2}
$$

which is now convex in the gradient and satisfies the assumptions of [30].

3.2. The ergodic problem and the effective Hamiltonian. For $\delta>0$, we consider the approximate ergodic problem

$$
\delta w_{\delta}+F\left(\bar{x}, y, \bar{p}, D w_{\delta}, D^{2} w_{\delta}\right)-|\sigma(\bar{x}, y) \bar{p}|^{2}=0,
$$

where

$$
F(\bar{x}, y, \bar{p}, q, Y):=-\operatorname{tr}\left(\tau \tau^{T}(y) Y\right)-\left|\tau^{T}(y) q\right|^{2}-b(y) \cdot q-2\left(\tau(y) \sigma^{T}(\bar{x}, y) \bar{p}\right) \cdot q .
$$

Under our standing assumptions we have the following results.

Proposition 3.2. Let assumption (E) hold. For any $(\bar{x}, \bar{p})$ fixed, there exists a unique solution $w_{\delta} \in C^{2}\left(\mathbb{R}^{m}\right)$ of (3.5) satisfying

$$
-\frac{1}{\delta} \inf _{y \in \mathbb{R}^{m}}\left|\sigma(\bar{x}, y)^{T} \bar{p}\right|^{2} \leq w_{\delta}(y) \leq \frac{1}{\delta} \sup _{y \in \mathbb{R}^{m}}\left|\sigma(\bar{x}, y)^{T} \bar{p}\right|^{2},
$$

such that

$$
\lim _{\delta \rightarrow 0} \delta w_{\delta}(y)=\text { const }:=\bar{H}(\bar{x}, \bar{p}) \text { locally uniformly. }
$$

Moreover $\bar{H}(\bar{x}, \bar{p})$ is the unique constant such that $(3.1)$ has a solution $w \in C^{2}\left(\mathbb{R}^{m}\right)$ satisfying

$$
|w(y)| \leq \bar{C}\left(1+\log \left(\sqrt{|y|^{2}+1}\right)\right) \quad \text { for all } y \in \mathbb{R}^{m}
$$

Finally $w$ is the unique (up to and additive constant) solution to (3.1) for $\lambda=\bar{H}(\bar{x}, \bar{p})$.

Remark 3.2. The growth estimate (3.8) implies that $w$ solution of (3.1) belongs to the class $\Phi$ defined in (3.2), allowing us to apply Proposition 3.1 and deriving the uniqueness of $\bar{H}$. Note that (3.8) is stronger than the growth required in $\Phi$, in particular it would be enough to prove (3.8) with a linear function of $y$ in the right-hand side. 
First, we prove the following local gradient bound for the solution of the $\delta$-ergodic problem.

Lemma 3.3. Let $\delta>0$ and $w_{\delta} \in C^{2}\left(\mathbb{R}^{m}\right)$ be the unique bounded solution of (3.5). Then for all $k \in \mathbb{N}$ and $\bar{x}, \bar{p} \in \mathbb{R}^{n}$, there exists $C>0$ such that it holds

$$
\max _{y \in \bar{B}_{k}}\left|D_{y} w_{\delta}(y ; \bar{x}, \bar{p})\right| \leq C,
$$

where $B_{k}$ is the ball with radius $k$ and center 0 and $C$ depends on $k$ and $\bar{p}$.

Proof. We refer to [7] where we proved the result by the Bernstein method under the assumption of periodicity; the extension to a local bound follows by cut-off functions arguments, following the derivation of similar estimates in [24]. We refer also to [33], Lemma 2.4 for an analogous result. We only note that a key role is played by the coercivity in the gradient of the ergodic problem, more precisely by the quadratic term in the gradient $\left|\tau^{T} D w_{\delta}\right|^{2}$.

Now we prove Proposition 3.2.

Proof of Proposition 3.2. We split the proof into two steps. In step 1 we prove the existence of a couple $(w, \lambda) \in C\left(\mathbb{R}^{m}\right) \times \mathbb{R}$ solution to (3.1); in step 2 we prove that $w \in C^{2}\left(\mathbb{R}^{m}\right)$, (3.8) and the uniqueness of such $\lambda$. Note that the uniqueness up to an additive constant of $w$ follows from Theorem 2.2 of [30].

Step. 1-Existence We use the methods of [5] based on the small discount approximation (3.5). Note that the PDE (3.5) has bounded forcing term $\left|\sigma^{T}(\bar{x}, y) \bar{p}\right|^{2}$ since $\sigma$ is bounded. The existence and uniqueness of a viscosity solution with the $\delta$ dependent bound (3.7) follows from the Perron-Ishii method and the comparison principle in [21]. Moreover $w_{\delta} \in$ $C^{2}\left(\mathbb{R}^{m}\right)$, thanks to the Lipschitz uniform estimate of Lemma 3.3 and by elliptic regularity theory of convex uniformly elliptic equations, see [43] and [42].

Now we prove that $\delta w_{\delta}(y)$ converges along a subsequence of $\delta \rightarrow 0$ to the constant $\bar{H}(\bar{x}, \bar{p})$ and $w_{\delta}(y)-w_{\delta}(0)$ converges to the corrector $w$. The hard part is proving equicontinuity estimates for $\delta w_{\delta}$. We proceed by a diagonal argument. By the local Lipschitz estimates of Lemma 3.3, we have

$$
\left|w_{\delta}(y)-w_{\delta}(z)\right| \leq C_{1}|y-z| \quad y, z \in \bar{B}_{1},
$$

where for convenience we denote by $C_{k}$ the constant of Lemma 3.3 in $B_{k}$ for $k \in \mathbb{N}$. Then $\delta w_{\delta}$ is equicontinuous in $\bar{B}_{1}$. The equiboundedness follows from the comparison principle with constant sub and super solutions, namely $\min _{y \in \mathbb{R}^{m}}\left|\sigma(y, \bar{x})^{T} \bar{p}\right|^{2}$ and $\max _{y \in \mathbb{R}^{m}}\left|\sigma(y, \bar{x})^{T} \bar{p}\right|^{2}$. Then by Ascoli-Arzela theorem, there exists a subsequence $\delta_{n}^{1} w_{\delta_{n}^{1}}$ of $\delta w_{\delta}$, converging uniformly in $\bar{B}_{1}$ to a constant $\lambda^{1}$, since by (3.10) we have

$$
\left|\delta w_{\delta}(y)-\delta w_{\delta}(z)\right| \leq \delta C_{1}|y-z| \quad y, z \in \bar{B}_{1}
$$

and then

$$
\delta w_{\delta}(y)-\delta w_{\delta}(z) \rightarrow 0 \quad \forall y, z \in \bar{B}_{1} \text { as } \delta \rightarrow 0 .
$$

By the same argument, $\delta_{n}^{1} w_{\delta_{n}^{1}}$ is equibounded and equicontinuous in $\bar{B}_{2}$. Then, there exists a subsequence $\delta_{n}^{2} w_{\delta_{n}^{2}}$ of $\delta_{n}^{1} w_{\delta_{n}^{1}}$, converging uniformly in $\bar{B}_{2}$ to a constant $\lambda^{2}$, such that

$$
\lambda^{1}=\lambda^{2}=: \lambda .
$$

Similarly, we construct for all $k \in \mathbb{N}$, a sequence $\left\{\delta_{n}^{k} w_{\delta_{n}^{k}}\right\}_{n}$ converging as $n \rightarrow \infty$ uniformly in $\bar{B}_{k}$ to a constant $\lambda^{k}=\lambda$. Note that the subsequence $\left\{\delta_{n}^{n} w_{\delta_{n}^{n}}\right\}_{n}$ converges locally uniformly to $\lambda$. In fact for any $k \in \mathbb{N}$ we have that $\left\{\delta_{n}^{n} w_{\delta_{n}^{n}}\right\}_{n}$ is a subsequence of $\left\{\delta_{n}^{k} w_{\delta_{n}^{k}}\right\}_{n}$ for all $n \geq k$, from which we deduce that $\left\{\delta_{n}^{n} w_{\delta_{n}^{n}}\right\}_{n}$ converges uniformly in $\bar{B}_{k}$ for all $k \in \mathbb{N}$. 
Now define $v_{\delta}:=w_{\delta}(y)-w_{\delta}(0)$. Notice that, for all $k, v_{\delta}$ is equibounded in $\bar{B}_{k}$, since, by Lemma 3.3, we have

$$
\left|v_{\delta}(y)\right|=\left|w_{\delta}(y)-w_{\delta}(0)\right| \leq C_{k}|y|, \quad y \in \bar{B}_{k}
$$

and, again by Lemma $3.3, v_{\delta}$ is equicontinuous in $B_{k}$ since

$$
\left|v_{\delta}(y)-v_{\delta}(z)\right|=\left|w_{\delta}(y)-w_{\delta}(z)\right| \leq C_{k}|y-z|, \quad y, z \in \bar{B}_{k} .
$$

By an analogous diagonal argument, we find sequences $\left\{v_{\delta_{n}^{k}}\right\}_{n}$ such that $v_{\delta_{n}^{k+1}}$ is a subsequence of $v_{\delta_{n}^{k}}$, and converges uniformly in $\bar{B}_{k}$ to a function $v^{k}$. Moreover for all $k \in \mathbb{N}$, we have

$$
v^{k+1}(y)=v^{k}(y) \quad y \in \bar{B}_{k} .
$$

Then, if we define $w: \mathbb{R}^{m} \rightarrow \mathbb{R}$ such that

$$
w(y)=v^{k}(y) \quad y \in \bar{B}_{k},
$$

we conclude that

$$
\left\{v_{\delta_{n}^{n}}\right\}_{n} \rightarrow w \text { locally uniformly. }
$$

Now we prove that $(\lambda, w)$ satisfy (3.1). From (3.5) we get

$$
\delta v_{\delta}+\delta w_{\delta}(0)+F\left(\bar{x}, y, \bar{p}, D_{y} v_{\delta}, D_{y y}^{2} v_{\delta}\right)-\left|\sigma^{T}(\bar{x}, y) \bar{p}\right|^{2}=0, \quad \text { in } \mathbb{R}^{m} .
$$

Since $v_{\delta}$ is locally equibounded, $\delta v_{\delta} \rightarrow 0$ locally uniformly and the claim follows recalling that $\delta w_{\delta} \rightarrow \lambda$ and using the stability property of viscosity solutions.

Finally the corrector inherits the property (3.9) of Lemma 3.3, that is, for all $k \in \mathbb{N}$ and (barx, $\bar{p}) \in \mathbb{R}^{n} \times \mathbb{R}^{n}$, there exists $C>0$ such that

$$
\max _{y \in \bar{B}_{k}}\left|D_{y} w(y ; \bar{x}, \bar{p})\right| \leq C,
$$

where $C$ depends on $k$ and $\bar{p}$.

Step. 2-Uniqueness of $\lambda$ The uniqueness is given by Proposition 3.1, once proved that $w \in \Phi$. The $C^{2}$ regularity follows from the uniform Lipschitz estimate (3.14) and the regularity theory of convex uniformly elliptic equations, see [43] and [42].

Note that, in order to prove that $w \in \Phi$, we prove the (stronger) growth condition (3.8). We prove the claim for the upper bound, since the proof of the lower bound is analogous.

We take the approximate problem (3.5) and we prove that the function $g=C \log \left(\sqrt{|y|^{2}+1}\right)$, for some positive constant $C$ large enough, is a supersolution of (3.5), that is, we prove

$$
\delta g(y)-\left(b(y)+2 \tau(y) \sigma^{T}(\bar{x}, y) \bar{p}\right) \cdot D g-\left|\tau^{T}(y) D g(y)\right|^{2}-\operatorname{tr}\left(\tau \tau^{T}(y) D^{2} g\right)-|\sigma(\bar{x}, y) \bar{p}|^{2} \geq 0 .
$$

Take $|y| \geq R$ where $R$ is defined in (E). By (E) and the boundedness of $\sigma$, we have

$$
\begin{array}{r}
\delta g(y)-\left(b(y)+2 \tau(y) \sigma^{T}(\bar{x}, y) \bar{p}\right) \cdot D g-\left|\tau^{T}(y) D g(y)\right|^{2}-\operatorname{tr}\left(\tau \tau^{T}(y) D^{2} g\right)-|\sigma(\bar{x}, y) \bar{p}|^{2} \geq \\
2 C B \frac{|y|^{2}}{|y|^{2}+1}-\frac{K C(1+|\bar{p}|)|y|}{|y|^{2}+1}-K C^{2} \frac{|y|^{2}}{\left(|y|^{2}+1\right)^{2}}-|\sigma(\bar{x}, y) \bar{p}|^{2}, \quad \text { (3.16) }
\end{array}
$$

where $K$ depends on $B>0$ defined in (E) and on $\|\sigma(\bar{x}, \cdot)\|_{\infty}$. Then, in order to prove that $g$ is a supersolution of (3.15), we prove that the right-hand side in (3.16) is non negative. We factorise $\frac{|y|^{2}}{|y|^{2}+1}$ and we prove that

$$
2 C B-\frac{K C(1+|\bar{p}|)}{|y|}-\frac{K C^{2}}{|y|^{2}+1}-\sup _{y}\left|\sigma^{T} \bar{p}\right|^{2} \frac{|y|^{2}+1}{|y|^{2}} \geq 0 .
$$


Note that when $y$ goes to infinity in (3.17) the leading order term is $2 C B-\sup _{y}\left|\sigma^{T} \bar{p}\right|^{2}$. Then the claim follows by taking $C$ such that $2 C B=2+\frac{3}{2} \sup _{y}\left|\sigma^{T} \bar{p}\right|^{2}$ and $y \in \mathbb{R}^{m} \backslash \bar{B}_{\bar{R}}$ for some $\bar{R}>R$ such that

$$
\frac{K C(1+|\bar{p}|)}{|y|}+\frac{K C^{2}}{|y|^{2}+1} \leq 2, \quad \frac{|y|^{2}+1}{|y|^{2}} \leq \frac{3}{2} .
$$

Up to now we proved that the function $C \log \left(\sqrt{|y|^{2}+1}\right)$ is a supersolution of (3.5) in $\mathbb{R}^{m} \backslash B_{\bar{R}}$. If $\max _{\bar{B}_{\bar{R}}} w_{\delta} \leq 0$ then

$$
w_{\delta}(y) \leq \max _{\bar{B}_{\bar{R}}} w_{\delta} \leq C \log \left(\sqrt{|y|^{2}+1}\right) \quad y \in \partial B_{\bar{R}},
$$

and then by the comparison principle we have

$$
w_{\delta}(y) \leq C \log \left(\sqrt{|y|^{2}+1}\right) \quad y \in \mathbb{R}^{m} .
$$

Now suppose that $\max _{\bar{B}_{\bar{R}}} w_{\delta} \geq 0$ and notice that in this case $C \log \left(\sqrt{|y|^{2}+1}\right)+\max _{\bar{B}_{\bar{R}}} w_{\delta}$ is still a supersolution of (3.5) in $\mathbb{R}^{m} \backslash B_{\bar{R}}$. Then, again by the comparison principle, we get

$$
w_{\delta}(y) \leq C \log \left(\sqrt{|y|^{2}+1}\right)+\max _{\bar{B}_{\bar{R}}} w_{\delta} \quad y \in \mathbb{R}^{m} .
$$

Since $w_{\delta}$ satisfies $(3.18)$

$$
v_{\delta}(y)=w_{\delta}(y)-w_{\delta}(0) \leq C \log \left(\sqrt{|y|^{2}+1}\right)+\max _{\bar{B}_{\bar{R}}} w_{\delta}(y)-w_{\delta}(0) \quad y \in \mathbb{R}^{m} .
$$

We estimate the term $\max _{\bar{B}_{\bar{R}}} w_{\delta}(y)-w_{\delta}(0)$ by Lemma 3.3 and we get

$$
v_{\delta}(y) \leq C \log \left(\sqrt{|y|^{2}+1}\right)+C_{\bar{R}}
$$

and thanks to (3.12) we conclude (3.8) by taking $\bar{C}=\max \left\{C, C_{\bar{R}}\right\}$.

We recall some properties satisfied by $\bar{H}$. For a proof we refer to [7], Proposition 3.3.

Proposition 3.4. Let assumption (E) hold.

(a) $\bar{H}$ is continuous on $\mathbb{R}^{n} \times \mathbb{R}^{n}$;

(b) the function $p \rightarrow \bar{H}(x, p)$ is convex;

(c)

$$
\inf _{y \in \mathbb{R}^{m}}\left|\sigma^{T}(\bar{x}, y) \bar{p}\right|^{2} \leq \bar{H}(\bar{x}, \bar{p}) \leq \sup _{y \in \mathbb{R}^{m}}\left|\sigma^{T}(\bar{x}, y) \bar{p}\right|^{2} ;
$$

(d) For all $0<\mu<1$ and $x, z, q, p \in \mathbb{R}^{n}$, it holds

$$
\mu \bar{H}\left(x, \frac{p}{\mu}\right)-\bar{H}(z, q) \geq \frac{1}{\mu-1} \sup _{y \in \mathbb{R}^{m}}\left|\sigma^{T}(x, y) p-\sigma^{T}(z, y) q\right|^{2} .
$$

Finally we observe that equations like (3.1) have been studied in a non compact setting by Khaise and Sheu in [33]. They prove the existence of a constant $\bar{H}$ such that there is a unique (up to an additive constant) smooth solution $w$ of (3.1) with prescribed growth. Moreover they provide a representation formula for $\bar{H}$ as the convex conjugate of a suitable operator over a space of measures. 


\section{The SUPERCRITICAL CASE: $\alpha>2$}

The ergodic problem is finding, for any $(\bar{x}, \bar{p}) \in \mathbb{R}^{n} \times \mathbb{R}^{n}$ fixed, a unique constant $\lambda \in \mathbb{R}$ such that the following uniformly elliptic linear equation has a viscosity solution $w$

$$
\lambda-\operatorname{tr}\left(\tau \tau^{T}(y) D^{2} w(y)\right)-b(y) \cdot D w(y)-\left|\sigma(\bar{x}, y)^{T} \bar{p}\right|^{2}=0 .
$$

Remark 4.1. Note that in (4.1) the correlation term $\tau(y) \sigma(\bar{x}, y)^{T} D w(y)$ does not arise, differently from the critical case see the ergodic problem (3.1). This suggests that the correlation between the asset and the volatility does not play any role when the volatility evolves very fast, that is, in the ultra-fast mean reverting regime.

This kind of ergodic problems has been studied in [6], see in particular Proposition 4.2 and Theorem 4.3.

Proposition 4.1. Let assumption (E) hold. For any $(\bar{x}, \bar{p}) \in \mathbb{R}^{n} \times \mathbb{R}^{n}$, there exists a unique invariant probability measure $\mu$ for the process

$$
d Y_{t}=b\left(Y_{t}\right) d t+\sqrt{2} \tau\left(Y_{t}\right) d W_{t} .
$$

Remark 4.2. For the details we refer to [6], Proposition 4.2. We just observe that the proof relies strongly on the existence of a Liapounov function as proved in the paragraph 2.3 for the infinitesimal generator of the process $(4.2)$, that is, the operator $\mathcal{L}(y, q, Y)=$ $\operatorname{tr}\left(\tau \tau^{T}(y) Y\right)-b(y) \cdot q$.

Consider the approximate $\delta$-ergodic problem for fixed $(\bar{x}, \bar{p}, \bar{X})$

$$
\delta w_{\delta}(y)-\left|\sigma(\bar{x}, y)^{T} \bar{p}\right|^{2}-b(y) \cdot D_{y} w_{\delta}(y)-\operatorname{tr}\left(\tau(y) \tau(y)^{T} D_{y y}^{2} w_{\delta}(y)\right)=0 \text { in } \mathbb{R}^{m} .
$$

We have the following proposition.

Proposition 4.2. Let assumption (E) and (S1) holds. For any fixed $(\bar{x}, \bar{p})$ there exists a unique solution $w_{\delta} \in C^{2}\left(\mathbb{R}^{m}\right)$ of (4.3) satisfying

$$
-\frac{1}{\delta} \inf _{y \in \mathbb{R}^{m}}\left|\sigma(\bar{x}, y)^{T} \bar{p}\right|^{2} \leq w_{\delta}(y) \leq \frac{1}{\delta} \sup _{y \in \mathbb{R}^{m}}\left|\sigma(\bar{x}, y)^{T} \bar{p}\right|^{2}
$$

such that

$$
\lim _{\delta \rightarrow 0} \delta w_{\delta}(y)=\int_{\mathbb{R}^{m}}\left|\sigma(\bar{x}, y)^{T} \bar{p}\right|^{2} d \mu(y):=\bar{H}(\bar{x}, \bar{p}) \text { locally uniformly },
$$

where $\mu$ is the unique invariant probability measure of the process (4.2). Moreover there exists a viscosity solution $w \in C^{2}\left(\mathbb{R}^{m}\right)$ of (4.1) with $\lambda=\bar{H}(\bar{x}, \bar{p})$ satisfying (3.8).

First we prove the following local gradient bound for the solution of the $\delta$-ergodic problem.

Lemma 4.3. Let (S1) hold. Let $\delta>0$ and $w_{\delta} \in C^{2}\left(\mathbb{R}^{m}\right)$ be the unique bounded solution of (4.3). Then for all $k \in \mathbb{N}$ and $\bar{x}, \bar{p} \in \mathbb{R}^{n}$, there exists $C>0$ such that it holds

$$
\max _{y \in \bar{B}_{k}}\left|D_{y} w_{\delta}(y ; \bar{x}, \bar{p})\right| \leq C,
$$

where $B_{k}$ is the ball of radius $k$ and center 0 and $C$ depends on $k$ and on $\bar{p}$.

Proof. We suppose that $\bar{p} \neq 0$, otherwise $w_{\delta}=0$ is the unique solution of (4.3). We observe that, by assumption (S1), if $\delta \leq \nu|\bar{p}|^{2}, 1$ is a subsolution of (4.3). Then, for such $\delta, w_{\delta} \geq 1$. Let $\bar{y}$ such that $w_{\delta}(\bar{y})=\min _{\bar{B}_{k}} w_{\delta}(y)$ and denote $M:=w_{\delta}(\bar{y})-1 \geq 0$. Let for $y \in \bar{B}_{k}$

$$
v_{\delta}(y)=\log \left(w_{\delta}(y)-M\right) \text {. }
$$


Then $v_{\delta}$ satisfies on $\bar{B}_{k}$

$$
\delta\left(1+e^{-v_{\delta}(y)} M\right)-\operatorname{tr}\left(\tau \tau^{T} D^{2} v_{\delta}(y)\right)-b \cdot D v_{\delta}(y)-\left|\tau^{T} D v_{\delta}(y)\right|^{2}-e^{-v_{\delta}(y)}\left|\sigma^{T} \bar{p}\right|^{2}=0
$$

and

$$
v_{\delta} \geq 0 \text { and } v_{\delta}(\bar{y})=0 .
$$

Note that for $y \in \bar{B}_{k}$, we have $1+e^{-v_{\delta}(y)} M \geq 0$ and $e^{-v_{\delta}(y)} \leq 1$. Then, by the coercivity of (4.8) and analogously to the critical case (see the proof of Lemma 3.3), we prove that there exists some positive constant $C$, depending on $k$ and $\bar{p}$, such that

$$
\max _{y \in \bar{B}_{k}}\left|D_{y} v_{\delta}(y ; \bar{x}, \bar{p})\right| \leq C .
$$

By (4.7) and (4.9), we have for $y \in \bar{B}_{k}$

$$
D w_{\delta}(y)=D v_{\delta}(y) e^{v_{\delta}(y)}=D v_{\delta}(y) e^{v_{\delta}(y)-v_{\delta}(\bar{y})}
$$

and, by (4.10), we finally get (4.6).

Proof of Proposition 4.2. For the identification of $\bar{H}$ and in particular for the proof of (4.5) we refer to [6], Theorem 4.3. For the existence of the corrector we note that the proof can be carried out analogously as in the critical case and we refer to the proof of Proposition 3.2 .

We observe that $\bar{H}$ satisfies the properties $(a),(b),(c),(d)$ of Proposition 3.4, which can be proved with similar arguments.

\section{Gradient Bounds}

In this section, we prove global uniform Lipschitz bounds for the solution of the approximate $\delta$-ergodic problems and of the true cell problems.

We recall that both $\|\tau\|_{\infty}$ and $\|\sigma\|_{\infty}$ are assumed finite.

The results are stated in the following propositions.

Proposition 5.1. Let assumptions (U), (S1) and (S2) hold. Let $w_{\delta} \in C^{2}\left(\mathbb{R}^{m}\right)$ be the unique bounded solution of (3.5) for $\alpha=2$ and of (4.3) for $\alpha>2$. Then for all $x, y \in \mathbb{R}^{m}$ we have

$$
\left|w_{\delta}(y ; \bar{x}, \bar{p})-w_{\delta}(x ; \bar{x}, \bar{p})\right| \leq C|x-y|
$$

where $C$ is a positive constant, depending on $\bar{x}, \bar{p},\|\tau\|_{\infty},\|\sigma\|_{\infty}, m$, the Lipschitz constants of $\tau, b, \sigma$ and is independent of $\delta$.

As a straightforward corollary of Proposition 5.1, we get the following global gradient bound for the correctors.

Proposition 5.2. Let assumptions (U), (S1) and (S2) hold. When $\alpha=2$ let $w \in C^{2}\left(\mathbb{R}^{m}\right)$ be a solution of (3.1) for $\lambda=\bar{H}(\bar{x}, \bar{p})$ where $\bar{H}(\bar{x}, \bar{p})$ is defined in Proposition 3.2; when $\alpha>2$ let $w \in C^{2}\left(\mathbb{R}^{m}\right)$ be the solution (defined in Proposition 4.2) of (4.1) for $\lambda=\bar{H}(\bar{x}, \bar{p})$. Then

$$
\sup _{y \in \mathbb{R}^{m}}\left|D_{y} w(y ; \bar{x}, \bar{p})\right| \leq C,
$$

where $C$ is a positive constant, depending on $\bar{x}, \bar{p},\|\tau\|_{\infty},\|\sigma\|_{\infty}, m$ and the Lipschitz constants of $\tau, b, \sigma$. 
The main result is Proposition 5.1. The proof is carried out in in two steps. In Step 1 we prove an Hölder bound not uniform in $\delta$ (see Proposition 5.3). The method is essentially based on the Ishii-Lions method and relies mainly on the uniform ellipticity of the equation. Then the result of Proposition 5.3 is used in Step 2 to complete the proof of Proposition 5.1. We remark that the result is non standard mainly because we do not use any compactness or periodicity of the coefficients, namely our result holds in all the space and is independent of $\delta$.

The proof of Proposition 5.3 and Proposition 5.1 are carried out only for $\alpha=2$ since the case $\alpha>2$ is analogous and even simpler.

Note that, thanks to the uniform local estimate previously proved in Lemma 3.3 for $\alpha=2$ and Lemma 4.3 for $\alpha>2$ (see respectively Section 3 and 4), the main difficulties come from the behaviour at infinity, which we treat by the assumptions (U) and (S2).

Step. 1-Global Hölder bounds

The proof of Proposition 5.3 is based on the Ishii-Lions method which allows us to take profit of the uniform ellipticity. As usual in the Ishii-Lions method, the estimate that we prove in (5.3) is not uniform in $\delta$. This is the main difference between Proposition 5.3 and Proposition 5.1 and, mainly for this reason, the proof of Proposition 5.3 is more standard.

Note that in the following proof we do not need assumptions (S1) and (S2), which, on the contrary, are fundamental in the proof of Proposition 5.1.

Proposition 5.3. Let assumption (U) hold. Let $w_{\delta} \in C^{2}\left(\mathbb{R}^{m}\right)$ be the unique bounded solution of (3.5) for $\alpha=2$ and of (4.3) for $\alpha>2$. Then there exists $C_{\delta}>0$ and $\alpha \in(0,1)$ such that

$$
\left|w_{\delta}(x ; \bar{x}, \bar{p})-w_{\delta}(y ; \bar{x}, \bar{p})\right| \leq C_{\delta}|x-y|^{\alpha} \quad \text { for all } x, y \in \mathbb{R}^{m},
$$

where $C_{\delta}$ depends on $\delta, \alpha,\|\tau\|_{\infty},\|\sigma(\bar{x}, \cdot)\|_{\infty}, \bar{p}$, the Lipschitz constants of $\tau, b, \sigma$ and $\theta$ of $(2.2)$.

Proof. We give the proof for $\alpha=2$ since the case $\alpha>2$ is analogous and even simpler.

Throughout the following proof we denote either by $(a, b)$ or $a \cdot b$ the scalar product for any $a, b \in \mathbb{R}^{m}$. For convenience of notation in the following we drop the dependence on $\bar{x}, \bar{p}$ by denoting the solution of $(3.5)$ by $w_{\delta}$.

Let $\delta>0$ and $\alpha \in(0,1)$ be fixed and consider the function

$$
w_{\delta}(x)-w_{\delta}(y)-C_{\delta}|x-y|^{\alpha},
$$

for some constant $C_{\delta}>0$ large enough. Note that $C_{\delta}$ will be chosen suitably at the end of the proof and will depend on $\delta, \alpha,\|\tau\|_{\infty},\|\sigma(\bar{x}, \cdot)\|_{\infty}, \bar{p}$, the Lipschitz constants of $\tau, b, \sigma$ and $\theta$ of (2.2). For clearness of exposition, we keep track only of the dependence on $\delta$.

We suppose that

$$
\sup \left\{w_{\delta}(x)-w_{\delta}(y)-C_{\delta}|x-y|^{\alpha}\right\}=M>0 .
$$

Let $R>0$ and consider the function

$$
\Phi(x, y)=w_{\delta}(x)-w_{\delta}(y)-C_{\delta}|x-y|^{\alpha}-\psi_{R}(x)-\psi_{R}(y)
$$

where

$$
\psi_{R}(z)=\psi\left(\frac{\sqrt{|z|^{2}+1}}{R}\right)
$$

and $\psi \in C^{2}([0,+\infty))$ satisfies

$$
\left\{\begin{array}{l}
\psi(s)=2\left\|w_{\delta}\right\|_{\infty}+1 \\
\psi(0)=0, \psi \geq 0, \psi^{\prime} \geq 0,
\end{array} \quad \text { if } s \geq 1\right.
$$


where we note that $\left\|w_{\delta}\right\|_{\infty}$ depends on $\delta$ as in (3.7). We claim that

$$
M_{R}=\sup \Phi(x, y) \rightarrow M \text { as } R \rightarrow+\infty \text {. }
$$

In fact

On the other hand

$$
M_{R} \leq M \quad \text { for any } R>0 .
$$

$$
M_{R} \geq w_{\delta}(x)-w_{\delta}(y)-C_{\delta}|x-y|^{\alpha}-\psi_{R}(x)-\psi_{R}(y) \text { for all } x, y \in \mathbb{R}^{m}, R>0,
$$

then

$$
\lim _{R \rightarrow+\infty} M_{R} \geq w_{\delta}(x)-w_{\delta}(y)-C_{\delta}|x-y|^{\alpha} \text { for all } x, y \in \mathbb{R}^{m}
$$

and we conclude

$$
\lim _{R \rightarrow+\infty} M_{R} \geq \sup \left\{w_{\delta}(x)-w_{\delta}(y)-C_{\delta}|x-y|^{\alpha}\right\}=M .
$$

Then we can suppose for $R$ large enough

$$
M_{R} \geq \frac{M}{2}>0 .
$$

We observe that if $\sqrt{|x|^{2}+1} \geq R$

$$
\Phi(x, y) \leq-1<0
$$

and the same holds when $\sqrt{|y|^{2}+1} \geq R$. Then, there exists $\left(x_{R}, y_{R}\right)$ point of maximum of $\Phi$ such that

$$
M_{R}=w_{\delta}\left(x_{R}\right)-w_{\delta}\left(y_{R}\right)-C_{\delta}\left|x_{R}-y_{R}\right|^{\alpha}-\psi_{R}\left(x_{R}\right)-\psi_{R}\left(y_{R}\right) .
$$

Note that $\left(x_{R}, y_{R}\right)$ depends also on $\delta$ and that we omit the dependence. Note also that

$$
\left|x_{R}-y_{R}\right|>0,
$$

otherwise by (5.9) we have

$$
M_{R}=-\psi_{R}\left(x_{R}\right)-\psi_{R}\left(y_{R}\right)
$$

and we get a contradiction by (5.8) and the definition of $\psi_{R}$.

By (??), (5.8) and the definition of $\psi_{R}$, we also have

$$
C_{\delta}\left|x_{R}-y_{R}\right|^{\alpha} \leq 2\left\|w_{\delta}\right\|_{\infty}:=A_{\delta} .
$$

Then

$$
\left|x_{R}-y_{R}\right| \leq\left(\frac{A_{\delta}}{C_{\delta}}\right)^{\frac{1}{\alpha}} .
$$

From now on we omit the dependence on $R$ and we write

$$
\left(x_{R}, y_{R}\right)=(x, y) \text {. }
$$

The main result is the following lemma.

Lemma 5.4. Under the above notations and assumption $(U)$, there exist positive constants $K, K_{1}, K_{2}, K_{3}, K_{4}$ such that

$$
\begin{array}{r}
0 \leq K C_{\delta} \alpha(\alpha-1)|x-y|^{\alpha-2}+K C_{\delta} \alpha|x-y|^{\alpha+1}+K_{1} C_{\delta} \alpha|x-y|^{\alpha}+K_{2} \alpha C_{\delta}^{2}|x-y|^{2 \alpha-1} \\
+K_{2} o_{R}(1) C_{\delta} \alpha|x-y|^{\alpha-1}+K_{3} \alpha C_{\delta}|x-y|^{\alpha-1}+K_{4}|x-y|+o_{R}(1) .
\end{array}
$$

where by $o_{R}(1)$ we mean that $o_{R}(1) \rightarrow 0$ as $R \rightarrow+\infty$. Moreover $K, K_{1}, K_{2}, K_{3}, K_{4}$ depends only on $\bar{p},\|\sigma\|_{\infty},\|\tau\|_{\infty}$, the Lipschitz constants of $\tau, b, \sigma$ and $\theta$ of $(2.2)$. 
Proof. Let

$$
r_{x}=D_{x} \psi_{R}=2 R^{-1} \psi^{\prime}\left(\frac{\sqrt{|x|^{2}+1}}{R}\right) x\left(\sqrt{|x|^{2}+1}\right)^{-1}
$$

and

$$
r_{y}=D_{y} \psi_{R}=2 R^{-1} \psi^{\prime}\left(\frac{\sqrt{|y|^{2}+1}}{R}\right) y\left(\sqrt{|y|^{2}+1}\right)^{-1}
$$

then for each $\delta$ fixed

$$
\left|r_{x}\right|,\left|r_{y}\right| \leq o_{R}(1), \quad\left\|D^{2} \psi_{R}\right\|_{\infty} \leq o_{R}(1)
$$

where $o_{R}(1)$ means that $\lim _{R \rightarrow+\infty} o_{R}(1)=0$.

We remark that in the rest of the proof we denote by $o_{R}(1)$ any function such that $o_{R}(1) \rightarrow 0$ as $R \rightarrow+\infty$. We also denote

$$
s=C_{\delta} \alpha|x-y|^{\alpha-2}(x-y) .
$$

Note that the function in (5.5) is smooth near $(x, y)$ by $(5.10)$. Then, since $w_{\delta}$ is a viscosity solution of $(3.5)$ and since $(x, y)$ is a maximum point of the function in $(5.5)$, we have

$$
\begin{aligned}
0 \leq \operatorname{tr}\left(\tau(x) \tau(x)^{T} D^{2} w_{\delta}(x)\right)-\operatorname{tr}\left(\tau(y) \tau(y)^{T} D^{2} w_{\delta}(y)\right) & +L(x, y)+G(x, y)+E(x, y) \\
& +F(x, y)+D(x, y)+o_{R}(1)
\end{aligned}
$$

where we used (5.14) to estimate the $\psi_{R}$-terms and we denoted

$$
\begin{gathered}
D(x, y)=\delta w_{\delta}(y)-\delta w_{\delta}(x) \\
L(x, y)=s \cdot(b(x)-b(y))+b(y) \cdot r_{y}+b(x) \cdot r_{x} \\
G(x, y)=\left|\tau(x)^{T}\left(s+r_{x}\right)\right|^{2}-\left|\tau(y)^{T}\left(s-r_{y}\right)\right|^{2} \\
E(x, y)=2 \tau(x) \sigma(\bar{x}, x)^{T} \bar{p} \cdot\left(s+r_{x}\right)-2 \tau(y) \sigma(\bar{x}, y)^{T} \bar{p} \cdot\left(s-r_{y}\right) ; \\
F(x, y)=\left|\sigma^{T}(\bar{x}, x) \bar{p}\right|^{2}-\left|\sigma^{T}(\bar{x}, y) \bar{p}\right|^{2} .
\end{gathered}
$$

First we estimate the second order terms in (5.16), by proving the following lemma.

Lemma 5.5. Under the above notations, we have

$$
\begin{aligned}
\operatorname{tr}\left(\tau(x) \tau(x)^{T} D^{2} w_{\delta}(x)\right)-\operatorname{tr}\left(\tau(y) \tau(y)^{T} D^{2} w_{\delta}(y)\right) \leq & K C_{\delta} \alpha(\alpha-1)|x-y|^{\alpha-2} \\
& +K C_{\delta} \alpha|x-y|^{\alpha+1}+o_{R}(1)
\end{aligned}
$$

where $K$ is a positive constant (depending on $\theta$ of (2.2) and on the Lipschitz constant of $\tau$ ) and by $o_{R}(1)$ we mean that $\lim _{R \rightarrow+\infty} o_{R}(1)=0$.

Proof. We observe that, for any orthonormal basis $e_{i}, i=1, \cdots m$ of $\mathbb{R}^{m}$, we can write

$$
\operatorname{tr}\left(\tau(x) \tau(x)^{T} D^{2} w_{\delta}(x)\right)=\sum_{i=1}^{m}\left(\tau(x) \tau(x)^{T} D^{2} w_{\delta}(x) e_{i}, e_{i}\right)=\sum_{i=1}^{m}\left(D^{2} w_{\delta}(x) \tau(x) e_{i}, \tau(x) e_{i}\right)
$$

Denote $\phi(t)=C_{\delta} t^{\alpha}, f(z)=|z|$. By the maximum point property and the second inequality of (5.14), we get

$$
\begin{aligned}
&\left(D^{2} w_{\delta}(x) p, p\right)-\left(D^{2} w_{\delta}(y) q, q\right) \leq \phi^{\prime}(f(x-y))\left(D^{2} f(x-y)(p-q),(p-q)\right) \\
&+\phi^{\prime \prime}(f(x-y))(D f(x-y), p-q)^{2}+o_{R}(1)
\end{aligned}
$$


for any $p, q \in \mathbb{R}^{m}$.

Next we remark that $|D f|^{2}=1$ and therefore, by differentiating this identity, we have $D^{2} f D f=0$. By $(2.2)$, we can set

$$
e_{1}=\frac{\tau(x)^{-1} D f(x-y)}{\left|\tau(x)^{-1} D f(x-y)\right|}, \quad \tilde{e}_{1}=-\frac{\tau(y)^{-1} D f(x-y)}{\left|\tau(y)^{-1} D f(x-y)\right|} .
$$

If $e_{1}, \tilde{e}_{1}$ are collinear, the we complete the basis with orthogonal unit vectors $e_{i}=\tilde{e}_{i} \in$ $e_{1}^{\top}, 2 \leq i \leq m$. Otherwise, in the plane $\operatorname{span}\left\{e_{1}, \tilde{e}_{1}\right\}$, we consider a rotation $\mathcal{R}$ of angle $\frac{\pi}{2}$ and we define

$$
e_{2}=\mathcal{R} e_{1}, \quad \tilde{e}_{2}=-\mathcal{R} \tilde{e}_{1} .
$$

Since $\operatorname{span}\left\{e_{1}, e_{2}\right\}^{\top}=\operatorname{span}\left\{\tilde{e}_{1}, \tilde{e}_{2}\right\}^{\top}$, we can complete the orthonormal basis with unit vectors $e_{i}=\tilde{e}_{i} \in \operatorname{span}\left\{e_{1}, e_{2}\right\}^{\top}, 3 \leq i \leq m$.

By (2.2), we have

Define

$$
\theta \leq \frac{1}{\left|\tau(x)^{-1} D f(x-y)\right|^{2}} \leq\|\tau\|_{\infty}^{2}
$$

$$
r_{1}=\tau(x) e_{1} \quad t_{1}=\tau(y) \tilde{e}_{1} .
$$

Since $|D f|=1$ and $D^{2} f D f=0$ and by choosing $p=r_{1}, q=r_{1}$ in (5.19), we get

$$
\begin{aligned}
\left(D^{2} w_{\delta}(x) r_{1}, r_{1}\right)-\left(D^{2} w_{\delta}(y) t_{1}, t_{1}\right) & \leq \phi^{\prime \prime}(f(x-y))\left(D f(x-y), r_{1}-t_{1}\right)^{2}+o_{R}(1) \\
= & C_{\delta} \alpha(\alpha-1)|x-y|^{\alpha-2}\left(D f(x-y), r_{1}-t_{1}\right)^{2}+o_{R}(1) .
\end{aligned}
$$

Notice that

$$
\alpha(\alpha-1)<0 .
$$

By (2.2), we have

Then

$$
\left(D f(x-y), r_{1}-t_{1}\right)^{2}=\left(\frac{1}{\left|\tau(x)^{-1} D f(x-y)\right|^{2}}+\frac{1}{\left|\tau(y)^{-1} D f(x-y)\right|}\right)^{2} \geq 4 \theta .
$$

$$
\left(D^{2} w_{\delta}(x) r_{1}, r_{1}\right)-\left(D^{2} w_{\delta}(y) t_{1}, t_{1}\right) \leq 4 \theta C_{\delta} \alpha(\alpha-1)|x-y|^{\alpha-2}+o_{R}(1) .
$$

Therefore in the right hand side we have a very negative term by a double effect, first because we will choose $C_{\delta}$ large but also because, by doing so, $|x-y|$ becomes smaller and smaller and $|x-y|^{\alpha-2}$ larger and larger.

Now we choose in (5.19) for all $i \in\{1, \cdots, m-1\}$

$$
p=\tau(x) e_{i} \quad q=\tau(y) \tilde{e}_{i} .
$$

Since $\tau$ is Lipschitz, we get

$$
\left(D^{2} w_{\delta}(x) \tau(x) e_{i}, \tau(x) e_{i}\right)-\left(D^{2} w_{\delta}(y) \tau(y) \tilde{e}_{i}, \tau(y) \tilde{e}_{i}\right) \leq K C_{\delta} \alpha|x-y|^{\alpha+1}+o_{R}(1),
$$

where $K$ depends on the Lipschitz constant of $\tau$. Then, by summing the previous equation on $i$ and adding (5.21), we get

$$
\begin{aligned}
\sum_{i=1}^{m}\left(D^{2} w_{\delta}(x) \tau(x) e_{i}, \tau(x) e_{i}\right)-\sum_{i=1}^{m}\left(D^{2} w_{\delta}(y) \tau(y) \tilde{e}_{i}, \tau \tilde{e}_{i}\right) \leq & K C_{\delta} \alpha(\alpha-1)|x-y|^{\alpha-2} \\
& +K C_{\delta} \alpha|x-y|^{\alpha+1}+o_{R}(1),
\end{aligned}
$$

when by $K$ we denote a constant depending on the Lipschitz constant of $\tau$ and on $\theta$. Then, by (5.18) with $e_{i}$ defined as above (and $\tilde{e}_{i}$ for $\operatorname{tr}\left(\tau(y) \tau(y)^{T} D^{2} w_{\delta}(y)\right)$ ), we finally get (5.17). 
Then (5.16) becomes

$$
\begin{aligned}
0 \leq K C_{\delta} \alpha(\alpha-1)|x-y|^{\alpha-2}+K C_{\delta} \alpha \mid x- & \left.y\right|^{\alpha+1}+L(x, y)+G(x, y) \\
& +E(x, y)+F(x, y)+D(x, y)+o_{R}(1)
\end{aligned}
$$

Finally we estimate the left terms $D, L, G, E, F$ in (5.22). First note that

$$
D(x, y)=\delta w_{\delta}(y)-\delta w_{\delta}(x) \leq 0
$$

First note that, by (5.15) and since $b$ is Lipschitz, we have

$$
L(x, y) \leq K_{1} C_{\delta} \alpha|x-y|^{\alpha}+b(y) \cdot r_{y}+b(x) \cdot r_{x} .
$$

where $K_{1}$ depends on the Lipschitz constant of $b$. Note that

$$
b(y) \cdot r_{y}+b(x) \cdot r_{x} \leq o_{R}(1) .
$$

Indeed, the previous inequality holds from the second of (5.14) when $x, y$ are uniformly bounded in $R$. Now suppose $|x| \rightarrow+\infty$ as $R \rightarrow+\infty$ (the argument being similar if $|y| \rightarrow+\infty)$. By assumption (U) we have

$$
b(x) \cdot r_{x}=(b-x) \cdot r_{x}
$$

and by (5.12), we have

$$
x \cdot r_{x}=2 R^{-1}|x|^{2} \psi^{\prime}\left(\frac{\sqrt{|x|^{2}+1}}{R}\right)\left(\sqrt{|x|^{2}+1}\right)^{-1}
$$

and since $\psi^{\prime} \geq 0$ by definition of $\psi_{R}$ we have

$$
x \cdot r_{x} \geq 0 .
$$

Then by (5.23) and (5.14), we get

$$
(b-x) \cdot r_{x} \leq o_{R}(1) .
$$

Then

$$
L(x, y) \leq K_{1} C_{\delta} \alpha|x-y|^{\alpha}+o_{R}(1) .
$$

Now we estimate the $G$-term. By the first of (5.14), (5.15) and since $\tau$ is bounded, we have

$$
G(x, y) \leq\left|\tau^{T}(x) s\right|^{2}-\left|\tau^{T}(y) s\right|^{2}+K_{2} o_{R}(1) C_{\delta} \alpha|x-y|^{\alpha-1}+o_{R}(1),
$$

where $K_{2}$ depends on $\|\tau\|_{\infty}$. Note that from now on we denote by $K_{2}$ a constant depending on $\|\tau\|_{\infty}$ and the Lipschitz constant of $\tau$ and which may change from line to line. Since $\tau$ is bounded by (5.15), we have

$$
\left|\tau^{T}(x) s\right|+\left|\tau^{T}(y) s\right| \leq K_{2} C_{\delta} \alpha|x-y|^{\alpha-1}
$$

and since $\tau$ is Lipschitz and by (5.15), we have

$$
\left|\tau^{T}(x) s\right|-\left|\tau^{T}(y) s\right| \leq K_{2} C_{\delta} \alpha|x-y|^{\alpha} .
$$

Then we get

and we conclude

$$
\left|\tau^{T}(x) s\right|^{2}-\left|\tau^{T}(y) s\right|^{2} \leq K_{2} \alpha C_{\delta}^{2} \alpha|x-y|^{2 \alpha-1},
$$

$$
G(x, y) \leq K_{2} \alpha C_{\delta}^{2}|x-y|^{2 \alpha-1}+K_{2} o_{R}(1) C_{\delta} \alpha|x-y|^{\alpha-1}+o_{R}(1) .
$$

Next we estimate $E$ using the boundedness of $\sigma$ and we get

$$
E(x, y) \leq K_{3} \alpha C_{\delta}|x-y|^{\alpha-1}+o_{R}(1),
$$

where $K_{3}>0$ depends on $\bar{p},\|\tau\|_{\infty},\|\sigma\|_{\infty}$. 
Finally, by the Lipschitz continuity and boundedness of $\sigma$, we have

$$
F(x, y) \leq K_{4}|x-y|,
$$

where $K_{4}$ depends on $\|\sigma\|_{\infty}$ and the Lipschitz constant of $\sigma$ and on $\bar{p}$.

Then, by all the previous estimates, (5.22) becomes

$$
\begin{aligned}
0 \leq K C_{\delta} \alpha(\alpha-1)|x-y|^{\alpha-2}+K C_{\delta} \alpha|x-y|^{\alpha+1}+K_{1} C_{\delta} \alpha|x-y|^{\alpha}+K_{2} \alpha C_{\delta}^{2}|x-y|^{2 \alpha-1} \\
+K_{2} o_{R}(1) C_{\delta} \alpha|x-y|^{\alpha-1}+K_{3} \alpha C_{\delta}|x-y|^{\alpha-1}+K_{4}|x-y|+o_{R}(1)
\end{aligned}
$$

This concludes the proof of Lemma 5.4.

We divide (5.25) by $C_{\delta}|x-y|^{\alpha-2}$ and we get

$$
\begin{array}{r}
0 \leq K \alpha(\alpha-1)+K \alpha|x-y|^{3}+K_{1} \alpha|x-y|^{2}+K_{2} \alpha C_{\delta}|x-y|^{\alpha+1}+K_{2} o_{R}(1) \alpha|x-y| \\
+K_{3} \alpha|x-y|+K_{4} C_{\delta}^{-1}|x-y|^{3-\alpha}+o_{R}(1) C_{\delta}^{-1}|x-y|^{2-\alpha} .
\end{array}
$$

Note that by (5.11), we have

then

$$
|x-y| \leq A_{\delta}^{\frac{1}{\alpha}} C_{\delta}^{-\frac{1}{\alpha}}
$$

$$
\begin{aligned}
& C_{\delta}^{-1}|x-y|^{3-\alpha} \leq A_{\delta}^{\frac{3-\alpha}{\alpha}} C_{\delta}^{-\frac{3}{\alpha}} ; \\
& C_{\delta}^{-1}|x-y|^{2-\alpha} \leq A_{\delta}^{\frac{2-\alpha}{\alpha}} C_{\delta}^{-\frac{2}{\alpha}} \\
& C_{\delta}|x-y|^{\alpha+1} \leq A_{\delta}^{\frac{\alpha+1}{\alpha}} C_{\delta}^{-\frac{1}{\alpha}} .
\end{aligned}
$$

By all the previous estimates and by taking $R$ large enough such that $o_{R}(1) \leq 1,(5.26)$ becomes

$$
\begin{aligned}
0 \leq K \alpha(\alpha-1)+K \alpha A_{\delta}^{\frac{3}{\alpha}} C_{\delta}^{-\frac{3}{\alpha}} & +K_{1} \alpha A_{\delta}^{\frac{2}{\alpha}} C_{\delta}^{-\frac{2}{\alpha}}+K_{2} \alpha A_{\delta}^{\frac{\alpha+1}{\alpha}} C_{\delta}^{-\frac{1}{\alpha}}+K_{2} o_{R}(1) \alpha A_{\delta}^{\frac{1}{\alpha}} C_{\delta}^{-\frac{1}{\alpha}} \\
& +K_{3} \alpha A_{\delta}^{\frac{1}{\alpha}} C_{\delta}^{-\frac{1}{\alpha}}+K_{4} A_{\delta}^{\frac{3-\alpha}{\alpha}} C_{\delta}^{-\frac{3}{\alpha}}+o_{R}(1) A_{\delta}^{\frac{2-\alpha}{\alpha}} C_{\delta}^{-\frac{2}{\alpha}}
\end{aligned}
$$

Then, the claim of the proposition follows by taking $C_{\delta}$ in (5.4) large enough in order to get a contradiction with (5.28). For example we take $C_{\delta}>\bar{C}_{\delta}$ where $\bar{C}_{\delta}$ satisfies

$$
\begin{aligned}
K \alpha(\alpha-1)+K \alpha A_{\delta}^{\frac{3}{\alpha}} \bar{C}_{\delta}^{-\frac{3}{\alpha}}+ & K_{1} \alpha A_{\delta}^{\frac{2}{\alpha}} \bar{C}_{\delta}^{-\frac{2}{\alpha}}+K_{2} \alpha A_{\delta}^{\frac{\alpha+1}{\alpha}} \bar{C}_{\delta}^{-\frac{1}{\alpha}}+K_{2} o_{R}(1) \alpha A_{\delta}^{\frac{1}{\alpha}} \bar{C}_{\delta}^{-\frac{1}{\alpha}} \\
& +K_{3} \alpha A_{\delta}^{\frac{1}{\alpha}} \bar{C}_{\delta}^{-\frac{1}{\alpha}}+K_{4} A_{\delta}^{\frac{3-\alpha}{\alpha}} \bar{C}_{\delta}^{-\frac{3}{\alpha}}+o_{R}(1) A_{\delta}^{\frac{2-\alpha}{\alpha}} \bar{C}_{\delta}^{-\frac{2}{\alpha}}<0 .
\end{aligned}
$$

Note that $\bar{C}_{\delta}$ depends on $K_{i}, i=1,2,3,4$ and on $\delta, \alpha, K$.

Step. 2-Proof of Proposition 5.1

Proof. Note that, under the assumption (U), (3.5) reads for $|y|>R_{1}$

$$
\delta w_{\delta}+F\left(\bar{x}, y, \bar{p}, D w_{\delta}, D^{2} w_{\delta}\right)-|\sigma(\bar{x}, y) \bar{p}|^{2}=0,
$$

where

$$
F(\bar{x}, y, \bar{p}, q, Y):=-\operatorname{tr}\left(\tau \tau^{T} Y\right)-\left|\tau^{T} q\right|^{2}-(b-y, q)-\left(2 \tau \sigma^{T}(\bar{x}, y) \bar{p}, q\right) .
$$

Note also that throughout the following proof we denote either by $(a, b)$ or $a \cdot b$ the scalar product for any $a, b \in \mathbb{R}^{m}$.

Let $\bar{R}>R_{1}$ be large enough (which will be chosen suitably at the end of the proof) and take $C_{\bar{R}}$ the constant of Lemma 3.3 for $k=\bar{R}$. Then we have for all $x, y \in \bar{B}_{\bar{R}}$

$$
\left|w_{\delta}(x ; \bar{x}, \bar{p})-w_{\delta}(y ; \bar{x}, \bar{p})\right| \leq C_{\bar{R}}|x-y| .
$$


For convenience of notation in the following we drop the dependence on $\bar{x}, \bar{p}$ by denoting the solution of $(5.29)$ by $w_{\delta}$.

In this first part of the proof we proceed analogously as in the proof of Proposition 5.3. The new part of the proof starts from Lemma 5.6. We give a sketch and for all the details we refer to the beginning of the proof of Proposition 5.3.

We proceed by contradiction and we suppose that

$$
\sup \left\{w_{\delta}(x)-w_{\delta}(y)-C|x-y|\right\}=M>0,
$$

where $C$ is a positive constant large enough, that is $C>\max \left\{C_{\bar{R}}, C_{\bar{R}+1}\right\}$.

Let $R>0$ and consider the function

$$
\Phi(x, y)=w_{\delta}(x)-w_{\delta}(y)-C|x-y|-\psi_{R}(x)-\psi_{R}(y),
$$

where

$$
\psi_{R}(z)=\psi\left(\frac{\sqrt{|z|^{2}+1}}{R}\right)
$$

where $\psi$ is defined in (5.7). By standard argument (see also the proof of Proposition 5.3), we prove that

$$
M_{R}=\sup \Phi(x, y) \rightarrow M \text { as } R \rightarrow+\infty,
$$

then we can suppose for $R$ large enough

$$
M_{R} \geq \frac{M}{2}>0,
$$

and by definition of $\psi_{R}$ we get that, for $R$ large enough, there exist $\left(x_{R}, y_{R}\right)$ such that

$$
M_{R}=w_{\delta}\left(x_{R}\right)-w_{\delta}\left(y_{R}\right)-C\left|x_{R}-y_{R}\right|-\psi_{R}\left(x_{R}\right)-\psi_{R}\left(y_{R}\right) .
$$

Note also that

$$
\left|x_{R}-y_{R}\right|>0 .
$$

We prove the following lemma, whose result is essential in order to use assumption (U) in the rest of the proof.

Lemma 5.6. Under the above notations, we have that, for $R$ large enough, there exists a point of maximum $\left(x_{R}, y_{R}\right)$ of the function $\Phi$ such that $\left(x_{R}, y_{R}\right) \in\left(\mathbb{R}^{m} \backslash \bar{B}_{\bar{R}}\right) \times\left(\mathbb{R}^{m} \backslash \bar{B}_{\bar{R}}\right)$. Moreover

$$
\liminf _{R \rightarrow+\infty}\left|x_{R}-y_{R}\right|>0 .
$$

Proof. Let $\left(x_{R}, y_{R}\right)$ be a point of maximum of $\Phi$ defined in (5.32) (see the above arguments for the existence). If $\left(x_{R}, y_{R}\right) \in\left(\mathbb{R}^{m} \backslash \bar{B}_{\bar{R}}\right) \times\left(\mathbb{R}^{m} \backslash \bar{B}_{\bar{R}}\right)$, the claim is proved. Otherwise, there are three possible cases (up to subsequences):

(i) $\left(x_{R}, y_{R}\right) \in \bar{B}_{\bar{R}} \times \bar{B}_{\bar{R}}$;

(ii) $\left(x_{R}, y_{R}\right) \in \bar{B}_{\bar{R}} \times\left(\mathbb{R}^{m} \backslash \bar{B}_{\bar{R}}\right)$;

(iii) $\left(x_{R}, y_{R}\right) \in\left(\mathbb{R}^{m} \backslash \bar{B}_{\bar{R}}\right) \times \bar{B}_{\bar{R}}$.

Suppose we are in case (i). We apply the local estimate on $\bar{B}_{\bar{R}}(5.30)$ and by the choice of $C$ in (5.31), we get a contradiction with (5.34).

Now we deal with case (ii) and we observe that case (iii) can be treated analogously. We prove that there exists $z_{R} \in \mathbb{R}^{m} \backslash \bar{B}_{\bar{R}}$ such that $\left(z_{R}, y_{R}\right)$ is still a maximum point of the function $\Phi$. Note that we can suppose that $y_{R} \in \mathbb{R}^{m} \backslash \bar{B}_{\bar{R}+1}$. Indeed, if $y_{R} \in \bar{B}_{\bar{R}+1}$, we use the local estimate on $\bar{B}_{\bar{R}+1}$ and by the choice of $C$ in (5.31), we get a contradiction with (5.34). Let $z_{R}, z_{R}^{\prime}$ be respectively the points where the segment between $x_{R}$ and $y_{R}$ intersects the boundary of $B_{\bar{R}+1}$ and of $B_{\bar{R}}$. Note that

$$
\left|x_{R}-y_{R}\right|=\left|x_{R}-z_{R}\right|+\left|z_{R}-y_{R}\right|
$$


and

$$
\left|x_{R}-z_{R}\right|=\left|x_{R}-z_{R}^{\prime}\right|+1 .
$$

Then, by (5.38), we have

$$
\max \Phi=\Phi\left(x_{R}, y_{R}\right) \leq \Phi\left(z_{R}, y_{R}\right)+w_{\delta}\left(x_{R}\right)-w_{\delta}\left(z_{R}\right)-C\left|x_{R}-z_{R}\right|-\psi_{R}\left(x_{R}\right)+\psi_{R}\left(z_{R}\right),
$$

and by the local estimate (5.30) on $\bar{B}_{\bar{R}+1}$ coupled with (5.39), we get

$$
\max \Phi \leq \Phi\left(z_{R}, y_{R}\right)+C_{\bar{R}+1}\left|x_{R}-z_{R}^{\prime}\right|+C_{\bar{R}+1}-C\left|x_{R}-z_{R}^{\prime}\right|-C-\psi_{R}\left(x_{R}\right)+\psi_{R}\left(z_{R}\right) .
$$

By the choice of $C$ in (5.31) we get

$$
\max \Phi \leq C_{\bar{R}+1}-C+\Phi\left(z_{R}, y_{R}\right)-\psi_{R}\left(x_{R}\right)+\psi_{R}\left(z_{R}\right)
$$

and, by taking $R$ large enough so that $C_{\bar{R}+1}-C-\psi_{R}\left(x_{R}\right)+\psi_{R}\left(z_{R}\right) \leq 0$, we conclude

$$
\max \Phi \leq \Phi\left(z_{R}, y_{R}\right) .
$$

Then, for $R$ large enough, $\left(z_{R}, y_{R}\right) \in\left(\mathbb{R}^{m} \backslash \bar{B}_{\bar{R}}\right) \times\left(\mathbb{R}^{m} \backslash \bar{B}_{\bar{R}}\right)$ is a point of maximum of the function $\Phi$. This conclude the proof of the first claim.

Now we prove (5.37). By contradiction, we suppose that

$$
\liminf _{R \rightarrow+\infty}\left|x_{R}-y_{R}\right|=0 .
$$

By (5.35) and the definition of $\psi_{R}$, we have

$$
M_{R} \leq w_{\delta}\left(x_{R}\right)-w_{\delta}\left(y_{R}\right) .
$$

Now we use Proposition 5.3 and by (5.3), we get

$$
M_{R} \leq C_{\delta}\left|x_{R}-y_{R}\right|^{\alpha} .
$$

Then, since $M_{R} \rightarrow M>0$, we get the following contradiction

$$
0<\liminf _{R \rightarrow+\infty} M_{R} \leq \liminf _{R \rightarrow+\infty} C_{\delta}\left|x_{R}-y_{R}\right|^{\alpha}=0,
$$

concluding the proof.

From now on we omit the dependence on $R$ and we write

$$
\left(x_{R}, y_{R}\right)=(x, y) \text {. }
$$

We prove the following lemma.

Lemma 5.7. Under the above notations and assumptions, there exists two positive constants $K_{1}, K_{2}$ such that

$$
C|x-y| \leq C K_{1} g(x, y)|x-y|+K_{2}|x-y|+o_{R}(1),
$$

where $g: \mathbb{R}^{m} \times \mathbb{R}^{m} \rightarrow \mathbb{R}^{+}$is such that $\forall \varepsilon>0$ there exists $R_{\varepsilon}$ such that $g(x, y) \leq \varepsilon$ for all $|x|,|y| \geq R_{\varepsilon}$. Moreover $K_{1}, K_{2}$ depends only on $\bar{p},\|\sigma\|_{\infty},\|\tau\|_{\infty}$ and by $o_{R}(1)$ we mean that $\lim _{R \rightarrow+\infty} o_{R}(1)=0$.

Remark 5.1. Note that $C|x-y|$, on the left side in (5.40), remains strictly positive for $R \rightarrow+\infty$ (by Lemma (5.6)). This term stems from the Ornstein-Uhlenbeck term $-(b-y) \cdot D w_{\delta}$ in the ergodic problem (5.29). 
Proof. We denote

$$
\begin{aligned}
& r_{x}:=D \psi_{R}(x)=R^{-1} \psi^{\prime}\left(\frac{\sqrt{|x|^{2}+1}}{R}\right) x\left(\sqrt{|x|^{2}+1}\right)^{-1} \\
& r_{y}:=D \psi_{R}(y)=R^{-1} \psi^{\prime}\left(\frac{\sqrt{|y|^{2}+1}}{R}\right) y\left(\sqrt{|y|^{2}+1}\right)^{-1} .
\end{aligned}
$$

We remark that

$$
\left|r_{x}\right|,\left|r_{y}\right| \leq R^{-1}|| \psi^{\prime} \|_{\infty}
$$

where $\left\|\psi^{\prime}\right\|_{\infty}$ depends on $\delta$. Similarly we argue for the second derivatives of $\psi_{R}$ and we get

$$
\left\|D^{2} \psi_{R}(z)\right\|_{\infty} \leq o_{R}(1)
$$

where $o_{R}(1)$ means that $\lim _{R \rightarrow+\infty} o_{R}(1)=0$.

Note that in the rest of the proof we denote by $o_{R}(1)$ any function respectively such that $o_{R}(1) \rightarrow 0$ as $R \rightarrow+\infty$. We also denote

$$
s=C \frac{x-y}{|x-y|} .
$$

Notice that the function in (5.32) is smooth since for $R$ big enough $x \neq y$ by Lemma 5.6. Then, since $w_{\delta}$ is a viscosity solution of $(5.29)$ and since $(x, y)$ is a maximum point of the function in (5.32), we have

$$
\begin{aligned}
L(x, y) \leq \operatorname{tr}\left(\tau \tau^{T} D^{2} w_{\delta}(x)\right)-\operatorname{tr}\left(\tau \tau^{T} D^{2} w_{\delta}(y)\right)+o_{R}(1)+G(x, y) & +E(x, y)+F(x, y)+D(x, y),
\end{aligned}
$$

where we used (5.43) and (5.44) to estimate the $\psi_{R}$-terms and where we denote

$$
\begin{gathered}
D(x, y)=\delta w_{\delta}(y)-\delta w_{\delta}(x) \\
L(x, y)=(s,(x-y))-\left(b-y, r_{y}\right)-\left(b-x, r_{x}\right) \\
G(x, y)=\left|\tau^{T}\left(s+r_{x}\right)\right|^{2}-\left|\tau^{T}\left(s-r_{y}\right)\right|^{2} \\
E(x, y)=\left(2 \tau \sigma(\bar{x}, x)^{T} \bar{p}, s+r_{x}\right)-\left(2 \tau \sigma(\bar{x}, y)^{T} \bar{p}, s-r_{y}\right) ; \\
F(x, y)=\left|\sigma^{T}(\bar{x}, x) \bar{p}\right|^{2}-\left|\sigma^{T}(\bar{x}, y) \bar{p}\right|^{2} .
\end{gathered}
$$

We estimate each term in (5.46). The most important terms is $L$ since it gives rise to the left order term $C|x-y|$ in (5.40). Indeed by (5.45), we have

$$
L(x, y) \geq C|x-y|-(\mu-y) \cdot r_{y}-(\mu-x) \cdot r_{x}
$$

and notice that by (5.41) and (5.42) we have

$$
x \cdot r_{x}=R^{-1}|x|^{2} \psi^{\prime}\left(\frac{\sqrt{|x|^{2}+1}}{R}\right)\left(\sqrt{|x|^{2}+1}\right)^{-1}
$$

and

$$
y \cdot r_{y}=R^{-1}|y|^{2} \psi^{\prime}\left(\frac{\sqrt{|y|^{2}+1}}{R}\right) y\left(\sqrt{|y|^{2}+1}\right)^{-1}
$$

and since $\psi^{\prime} \geq 0$ by definition of $\psi_{R}$, we have

$$
x \cdot r_{x} \geq 0, \quad y \cdot r_{y} \geq 0 .
$$

By (5.47) and (5.43), we get

$$
-(b-y) \cdot r_{y}-(b-x) \cdot r_{x} \geq o_{R}(1),
$$


and then

Then by the previous estimates we get

$$
L(x, y) \geq C|x-y|+o_{R}(1) .
$$

$C|x-y| \leq \operatorname{tr}\left(\tau \tau^{T} D^{2} w_{\delta}(x)\right)-\operatorname{tr}\left(\tau \tau^{T} D^{2} w_{\delta}(y)\right)+o_{R}(1)+G(x, y)+E(x, y)+F(x, y)+D(x, y)$.

Now we estimate the remaining terms in the right-hand side of (5.48). First note that

$$
D(x, y)=\delta w_{\delta}(y)-\delta w_{\delta}(x) \leq 0 .
$$

By (5.43) and (5.45), we have

$$
G(x, y) \leq o_{R}(1) .
$$

Next, by (S1) and (S2) and the boundedness of $\sigma$, we have

$$
E(x, y) \leq C K_{1} g(x, y)|x-y|+o_{R}(1),
$$

where $K_{1}>0$ depends on $\bar{p},\|\tau\|_{\infty},\|\sigma\|_{\infty}$.

By the Lipschitz continuity and boundedness of $\sigma$, we have

$$
F(x, y) \leq K_{2}|x-y|,
$$

where $K_{2}$ depends on $\|\sigma\|_{\infty}$ and the Lipschitz constant of $\sigma$ and on $\bar{p}$.

Finally we estimate the second order terms in (5.48) as follows

$$
\operatorname{tr}\left(\tau \tau^{T} D^{2} w_{\delta}(x)\right)-\operatorname{tr}\left(\tau \tau^{T} D^{2} w_{\delta}(y)\right) \leq o_{R}(1) .
$$

where by $o_{R}(1)$ we mean that $\lim _{R \rightarrow+\infty} o_{R}(1)=0$. The proof of (5.50) is analogous to the proof of (5.17), Lemma 5.5, Proposition 5.3 and even simpler. Indeed, we use again the following property: if $e_{i}, i=1, \cdots m$ is an orthonormal basis of $\mathbb{R}^{m}$ and $A$ is a matrix $m \times m$, we have

$$
\operatorname{tr}(A)=\sum_{i=1}^{m}\left(A e_{i}, e_{i}\right)
$$

then for any orthonormal basis $e_{i}, i=1, \cdots m$ of $\mathbb{R}^{m}$, we can write

$$
\operatorname{tr}\left(\tau \tau^{T} D^{2} w_{\delta}(x)\right)=\sum_{i=1}^{m}\left(\tau \tau^{T} D^{2} w_{\delta}(x) e_{i}, e_{i}\right)=\sum_{i=1}^{m}\left(D^{2} w_{\delta}(x) \tau e_{i}, \tau e_{i}\right) .
$$

Denote $f(z)=|z|$. We recall that the function in (5.32) is smooth at $(x, y)=\left(x_{R}, y_{R}\right)$ for $R$ large enough by Lemma 5.6. Then, since $x, y$ is a maximum point of the function in (5.32) and by (5.44), we get

$$
\left(D^{2} w_{\delta}(x) p, p\right)-\left(D^{2} w_{\delta}(y) q, q\right) \leq C\left(D^{2} f(x-y)(p-q),(p-q)\right)+o_{R}(1)
$$

for any $p, q \in \mathbb{R}^{m}$. Then, in order to prove the claim, it is enough to choose in (5.52) for all $i \in\{1, \cdots m\}$

Then we get

$$
p=\tau e_{i}, \quad q=\tau e_{i}
$$

$$
\left(D^{2} w_{\delta}(x) \tau e_{i}, \tau e_{i}\right)-\left(D^{2} w_{\delta}(y) \tau e_{i}, \tau e_{i}\right) \leq o_{R}(1) \text { for all } i \in\{1, \cdots m\},
$$

and by summing the previous equation on $i$, we get

$$
\sum_{i=1}^{m}\left(D^{2} w_{\delta}(x) \tau e_{i}, \tau e_{i}\right)-\sum_{i=1}^{m}\left(D^{2} w_{\delta}(y) \tau e_{i}, \tau e_{i}\right) \leq o_{R}(1)
$$

from which we conclude (5.50). By coupling all the previous estimates, we get (5.40) and we conclude the proof of Lemma 5.7. 
Now we conclude the the argument as follows. We use assumptions (S1) and (S2) and by taking $\bar{R}>R_{1}$ large enough, we consider $|x|,|y|$ large enough, such that

$$
K_{1} g(x, y) \leq \frac{1}{2} .
$$

Now we send $R \rightarrow+\infty$ in (5.40) and divide by $|x-y|$ thanks to Lemma 5.6, and we get

$$
C \leq \frac{C}{2}+K_{2}
$$

Then, to get a contradiction with (5.54), it is enough to take $C$ large enough such that

$$
C>2 K_{2} \text {. }
$$

Note that $C, \bar{R}$ depend respectively only on $K_{2}, R_{1}$ and in particular, they are independent on $\delta$.

Then the proof follows by taking $C$ in (5.31), such that $C>\max \left\{C_{\bar{R}}, C_{\bar{R}+1}, 2 K_{2}\right\}$, where $\bar{R}>R_{1}$ is such that (5.53) holds.

\section{THE COMPARISON PRINCIPLE}

In this section we provide the comparison principle for the limit PDE

$$
v_{t}-\bar{H}(x, D v)=0 \quad \text { in }(0, T) \times \mathbb{R}^{n},
$$

where $\bar{H}$ is defined in Proposition 3.2 for $\alpha=2$ and in Proposition 4.2 for $\alpha>2$.

Note that the comparison principle for the limit problem is a crucial ingredient in the proof of the convergence, which we address in the following section.

Theorem 6.1. Let assumption (U) hold. Let $u \in B U S C\left([0, T] \times \mathbb{R}^{n}\right)$ and $v \in B L S C([0, T] \times$ $\left.\mathbb{R}^{n}\right)$ be, respectively, a subsolution and a supersolution to (6.1) such that $u(0, x) \leq v(0, x)$ for all $x \in \mathbb{R}^{n}$. Then $u(x, t) \leq v(x, t)$ for all $x \in \mathbb{R}^{n}$ and $0 \leq t \leq T$.

Proof. The proof is exactly the same to [7] Theorem 3.5, in particular is based on the properties $(a),(b),(c),(d)$ of Proposition 3.4 satisfied by the effective Hamiltonian $\bar{H}$.

\section{The CONVERGence Result}

In this section we prove the convergence of the $v^{\varepsilon}$ to the unique solution of the limit problem (7.3). Throughout this section, let assumptions (U), (S1) and (S2) hold. Let $\alpha \geq 2$. We recall that $v^{\varepsilon}$ denotes the unique bounded viscosity solution of

$$
\begin{cases}\partial_{t} v^{\varepsilon}-H^{\varepsilon}\left(x, y, D_{x} v^{\varepsilon}, \frac{D_{y} v^{\varepsilon}}{\varepsilon^{\alpha-1}}, D_{x x}^{2} v^{\varepsilon}, \frac{D_{y y}^{2} v^{\varepsilon}}{\varepsilon^{\alpha-1}}, \frac{D_{x y}^{2} v^{\varepsilon}}{\varepsilon^{\frac{\alpha-1}{2}}}\right)=0 & \text { in }[0, T] \times \mathbb{R}^{n} \times \mathbb{R}^{m}, \\ v^{\varepsilon}(0, x, y)=h(x) & \text { in } \mathbb{R}^{n} \times \mathbb{R}^{m} .\end{cases}
$$

where

$$
\begin{aligned}
H^{\varepsilon}(x, y, p, q, X, Y, Z) & :=\left|\sigma^{T} p\right|^{2}+b \cdot q+\operatorname{tr}\left(\tau \tau^{T} Y\right)+\varepsilon\left(\operatorname{tr}\left(\sigma \sigma^{T} X\right)+\phi \cdot p\right) \\
& +2 \varepsilon^{\frac{\alpha}{2}-1}\left(\tau \sigma^{T} p\right) \cdot q+2 \varepsilon^{\frac{1}{2}} \operatorname{tr}\left(\sigma \tau^{T} Z\right)+\varepsilon^{\alpha-2}\left|\tau^{T} q\right|^{2} .
\end{aligned}
$$

We state and prove the convergence result. We will make use of the relaxed semi-limits which we define as follows. We denote

$$
\begin{aligned}
& v_{1}(t, x):=\inf \left\{\liminf _{\varepsilon \rightarrow 0}\left\{v^{\varepsilon}\left(t_{\varepsilon}, x_{\varepsilon}, y_{\varepsilon}\right) \mid x_{\varepsilon} \rightarrow x, t_{\varepsilon} \rightarrow t, y_{\varepsilon} \text { bounded }\right\}\right\}, \\
& v_{2}(t, x):=\sup \left\{\limsup _{\varepsilon \rightarrow 0}\left\{v^{\varepsilon}\left(t_{\varepsilon}, x_{\varepsilon}, y_{\varepsilon}\right) \mid x_{\varepsilon} \rightarrow x, t_{\varepsilon} \rightarrow t, y_{\varepsilon} \text { bounded }\right\}\right\},
\end{aligned}
$$


and we define the lower semi-limit as $\underline{v}=\left(v_{1}\right)_{*}$ the lower semicontinuous regularization of $v_{1}$ and the upper semi-limit as $\bar{v}=v_{2}^{*}$ the upper semicontinuous regularization of $v_{2}$. Since $h$ is bounded, the family $v^{\varepsilon}$ is equibounded and we have $\bar{v} \in B U S C\left([0, T] \times \mathbb{R}^{n}\right)$ and $\underline{v} \in B L S C\left([0, T] \times \mathbb{R}^{n}\right)$. Notice that by definition, we have

$$
\bar{v}(x, t) \geq \underline{v}(x, t) .
$$

Theorem 7.1. Let assumptions (U), (S1) and (S2) hold. Recall the effective problem

$$
v_{t}-\bar{H}(x, D v)=0 \text { in }(0, T) \times \mathbb{R}^{n} \quad v(0, x)=h(x) \text { on } \mathbb{R}^{n}
$$

where $\bar{H}$ is defined by Proposition 3.2 for $\alpha=2$ and Proposition 4.2 for $\alpha>2$. Then

a) the upper limit $\bar{v}$ of $v^{\varepsilon}$ is a subsolution of (7.3);

b) the lower limit $\underline{v}$ is a supersolution of (7.3);

c) $v^{\varepsilon}$ converges uniformly on the compact subsets of $[0, T) \times \mathbb{R}^{n} \times \mathbb{R}^{m}$ to the unique viscosity solution of (7.3).

Proof. Note that, once $a$ ) and $b$ ) proved, by the definition of semilimits and by the comparison principle (Theorem 6.1) for the effective equation (7.3), we get

$$
\bar{v}=\underline{v}=v \quad \text { in }[0, T) \times \mathbb{R}^{n}
$$

and then, thanks to the properties of semilimits, we get that $v^{\varepsilon}$ converges locally uniformly to the unique bounded solution of (7.3). Therefore, the main claims which we have to prove are $a$ ) and $b$ ). We prove only $a$ ) since the proof of $b$ ) is analogous. Moreover, since the proofs for the critical and supercritical case are similar with some minor (and standard) adaptations, we treat only the case $\alpha=2$.

We take a smooth function $\psi$, and without loss of generality we assume that $\psi$ is coercive in the variable $x$ and for all compact $K \subset[0, T] \times \mathbb{R}^{n}$ there exists a constant $C_{K}>0$ such that

$$
\left|\partial_{t} \psi(t, x)\right| \leq C_{K} \quad \forall(t, x) \in K .
$$

Let $(\bar{t}, \bar{x})$ be a point of strict maximum of $\bar{v}(t, x)-\psi(t, x)$. Let $\eta>0$ and consider the function

$$
\Phi(t, x, y)=v^{\varepsilon}(t, x, y)-\psi(t, x)-\varepsilon(w(y)+\eta \chi(y)),
$$

where $w$ is the corrector, solution to the ergodic problem (3.1) for $\lambda=\bar{H}\left(\bar{x}, D_{x} \psi(\bar{t}, \bar{x})\right)$ and $\chi$ is the Liapounov function, that is

$$
\chi=a|y|^{2}, \quad a<\frac{1}{2 T},
$$

for some $T>0$ depending on $\|\tau\|_{\infty}$ which we defined in (2.11).

By (3.8) and the definition (2.10) of $\chi$, we have for $\eta$ fixed

$$
w(y)+\eta \chi(y) \rightarrow+\infty \text { as }|y| \rightarrow+\infty .
$$

Then, there exists $\left(t_{\varepsilon, \eta}, x_{\varepsilon, \eta}, y_{\varepsilon, \eta}\right) \in[0, T] \times \mathbb{R}^{n} \times \mathbb{R}^{m}$ point of maximum of $\Phi$ defined in (7.5). We denote

$$
\left(t_{\varepsilon, \eta}, x_{\varepsilon, \eta}, y_{\varepsilon, \eta}\right)=:(t, x, y) .
$$

Since $v_{\varepsilon}$ is a solution of equation (7.1), we test it as a subsolution with the function $\psi+\varepsilon(w+\eta \chi)$ and by writing

$$
\left|\tau(y)^{T}(D w(y)+\eta D \chi(y))\right|^{2}=\left|\tau(y)^{T} D w(y)\right|^{2}+\eta^{2}\left|\tau(y)^{T} D \chi(y)\right|^{2}+2 \eta\left(\tau(y)^{T} D w(y), D \chi(y)\right),
$$


we get

$$
\begin{array}{r}
\psi_{t}(t, x)-\varepsilon \operatorname{tr}\left(\sigma \sigma(x, y)^{T} D_{x x}^{2} \psi(t, x)\right)-\varepsilon \phi(x, y) \cdot D_{x} \psi(t, x)-\left|\sigma(x, y)^{T} D_{x} \psi(t, x)\right|^{2} \\
-b(y) \cdot D w(y)-\operatorname{tr}\left(\tau(y) \tau(y)^{T} D^{2} w(y)\right)-2 \tau(y)^{T} \sigma(x, y)^{T} D_{x} \psi(t, x) \cdot D w(y)-\left|\tau(y)^{T} D w(y)\right|^{2} \\
+\eta G_{\varepsilon, \eta}(x, y) \leq 0, \quad(7.7)
\end{array}
$$

where, for convenience of notations, we denote

$$
\begin{aligned}
G_{\varepsilon, \eta}(x, y)=-b(y) \cdot D \chi(y)-\operatorname{tr}\left(\tau(y) \tau(y)^{T} D^{2} \chi(y)\right)-\eta\left|\tau(y)^{T} D \chi(y)\right|^{2} \\
-2 \tau(y)^{T} D w(y) \cdot D \chi(y)-2 \tau(y) \sigma(x, y)^{T} D_{x} \psi(t, x) \cdot D \chi(y) .
\end{aligned}
$$

We recall that the corrector $w$ is solution of the ergodic problem $(3.1)$ for $\lambda=\bar{H}\left(\bar{x}, D_{x} \psi(\bar{t}, \bar{x})\right)$ (see Proposition 3.2), that is, $w$ satisfies

$$
\begin{aligned}
\bar{H}(\bar{x}, D \psi(\bar{t}, \bar{x}))=b(y) & \cdot D w(y)+\operatorname{tr}\left(\tau(y) \tau(y)^{T} D^{2} w(y)\right)+\left|\tau(y)^{T} D w(y)\right|^{2} \\
& +2\left(\tau(y) \sigma(\bar{x}, y)^{T} D_{x} \psi(\bar{t}, \bar{x})\right) \cdot D w(y)+\left|\sigma(\bar{x}, y)^{T} D_{x} \psi(\bar{t}, \bar{x})\right|^{2} .
\end{aligned}
$$

We use (7.9) in (7.7) and we get

$$
\begin{array}{r}
\psi_{t}(t, x)-\varepsilon \operatorname{tr}\left(\sigma \sigma(x, y)^{T} D_{x x}^{2} \psi(t, x)\right)-\varepsilon \phi(x, y) \cdot D_{x} \psi(t, x)+\eta G_{\varepsilon, \eta}(x, y)+F_{\varepsilon}(x, y) \\
-\bar{H}(\bar{x}, D \psi(\bar{t}, \bar{x})) \leq 0,
\end{array}
$$

where we denote

$$
\begin{aligned}
F_{\varepsilon}(x, y)=\left(-2 \tau(y) \sigma(x, y)^{T} D_{x} \psi(t, x)\right. & \left.+2 \tau(y) \sigma(\bar{x}, y)^{T} D_{x} \psi(\bar{t}, \bar{x})\right) \cdot D w(y) \\
& -\left|\sigma(x, y)^{T} D_{x} \psi(t, x)\right|^{2}+\left|\sigma(\bar{x}, y)^{T} D_{x} \psi(\bar{t}, \bar{x})\right|^{2} .
\end{aligned}
$$

In the following lemma we prove that $(x, t, y)$ are uniformly bounded in $\varepsilon$ and that $x, t \rightarrow \bar{x}, \bar{t}$ as $\varepsilon \rightarrow 0$. Note that we split the proof of the equiboundedness of $(t, x, y)$ into (i) and (ii) in the following lemma only for convenience of exposition.

Lemma 7.2. Let $\eta>0$ be fixed. Under the above notations and under the assumptions of Theorem 7.1, we have

(i) $(x, t)$ are uniformly bounded in $\varepsilon$;

(ii) $y$ is uniformly bounded in $\varepsilon$;

(iii) $(x, t) \rightarrow(\bar{x}, \bar{t})$ as $\varepsilon \rightarrow 0$.

We split the proof into three steps; in Step 1 we prove (i), in Step 2 we prove (ii) and in Step 3 we prove (iii).

Proof of Lemma 7.2.

Step. 1 (Proof of (i)) For all $x^{\prime} \in \mathbb{R}^{n}, y^{\prime} \in \mathbb{R}^{m}$ and $t^{\prime} \in(0, T)$ we have

$$
v^{\varepsilon}(t, x, y)-\psi(t, x)-\varepsilon(w(y)+\eta \chi(y)) \geq v^{\varepsilon}\left(t^{\prime}, x^{\prime}, y^{\prime}\right)-\psi\left(t^{\prime}, x^{\prime}\right)-\varepsilon\left(w\left(y^{\prime}\right)+\eta \chi\left(y^{\prime}\right)\right),
$$

that is

$$
\psi(t, x)+\varepsilon(w(y)+\eta \chi(y)) \leq 2 \sup _{\varepsilon}\left\|v^{\varepsilon}\right\|_{\infty}+\sup _{\varepsilon}\left[\psi\left(t^{\prime}, x^{\prime}\right)+\varepsilon\left(w\left(y^{\prime}\right)+\eta \chi\left(y^{\prime}\right)\right)\right]
$$

then

$$
\left.\sup _{\varepsilon}[\psi(t, x)+\varepsilon(w(y)+\eta \chi(y)))\right]<\infty .
$$

Note that (7.12) implies

$$
\sup _{\varepsilon} \psi(t, x)<\infty
$$


Indeed, (7.13) follows immediately from (7.12) if $|y|$ is bounded in $\varepsilon$; when $|y| \rightarrow+\infty$ it follows since $\varepsilon(w(y)+\eta \chi(y)))$ is positive thanks to the definition of $\chi$ and the logarithmic growth of $w$ proved in (3.8). Then the uniform boundedness of $x$ and $t$ follows from (7.13) and the coercivity of $\psi$.

Step. 2 (Proof of (ii)) We proceed by contradiction, supposing $|y| \rightarrow+\infty$ as $\varepsilon \rightarrow 0$ and we get a contradiction with the equation (7.10) by applying Lemma 7.3, whose proof is postponed at the end of the proof of $a$ ). We just observe that it essentially relies on $(i)$ of Lemma 7.2 proved in step 1, on the quadratic growth of the Liapounov function $\chi$ and on the uniform estimate of the gradient of the corrector $w$ (Proposition 5.2).

Lemma 7.3. Let assumptions of Theorem 7.1 hold. Let $G_{\varepsilon, \eta}(x, y)$ and $F_{\varepsilon}(x, y)$ be defined respectively in (7.8) and (7.11) and let $\eta>0$ be fixed. Then, if

$$
|y| \rightarrow+\infty \text { as } \varepsilon \rightarrow 0
$$

then we have

(1) $\lim _{\varepsilon \rightarrow 0} G_{\varepsilon, \eta}(x, y)=+\infty$.

(2) $\left|\lim _{\varepsilon \rightarrow 0} F_{\varepsilon}(x, y)\right| \leq C^{\prime}$, for some constant $C^{\prime}>0$.

Then the uniform boundedness of $y$ follows by coupling (1) and (2) of Lemma 7.3 with equation (7.10) and observing that $\phi$ and $\sigma$ are bounded, $t, x$ are uniformly bounded in $\varepsilon$ and the time derivative of $\psi$ is bounded by (7.4).

Step. 3 (Proof of (iii)) Note that, by Step 1 and Step 2, we can suppose that there exists $(\tilde{t}, \tilde{x}, \tilde{y})$ such that, up to subsequences

$$
(t, x, y) \rightarrow(\tilde{t}, \tilde{x}, \tilde{y}) \quad \text { as } \varepsilon \rightarrow 0 .
$$

Since, for all $t^{\prime}, x^{\prime}, y^{\prime}$,

$$
v^{\varepsilon}(t, x, y)-\psi(t, x)-\varepsilon(w(y)+\eta \chi(y)) \geq v^{\varepsilon}\left(t^{\prime}, x^{\prime}, y^{\prime}\right)-\psi\left(t^{\prime}, x^{\prime}\right)-\varepsilon\left(w\left(y^{\prime}\right)-\eta \chi\left(y^{\prime}\right)\right),
$$

using the uniform boundedness of $y$ and the definition of upper semi-limit we get

$$
\bar{v}(\tilde{t}, \tilde{x})-\psi(\tilde{t}, \tilde{x}) \geq \bar{v}\left(t^{\prime}, x^{\prime}\right)-\psi\left(t^{\prime}, x^{\prime}\right) \quad \forall t^{\prime}, x^{\prime} .
$$

Then

$$
\tilde{x}=\bar{x}, \quad \tilde{t}=\bar{t}
$$

and

concluding the proof of the lemma.

$$
t \rightarrow \bar{t}, \quad x \rightarrow \bar{x} \quad \text { as } \varepsilon \rightarrow 0,
$$

Now we conclude the proof of Theorem $7.1 \mathrm{a}$ ).

Note that from now on when we do the limit as $\varepsilon \rightarrow 0$, we mean the limit along the subsequences such that (7.15) (and then also (7.16)) hold.

Note that, by $(\mathrm{iii})$ of Lemma 7.2 and by definition of the corrector $w$, we have

$$
\lim _{\varepsilon \rightarrow 0} F_{\varepsilon}(x, y)=0 \text {, }
$$

where $F_{\varepsilon}$ is defined in (7.11). Then, we let $\varepsilon \rightarrow 0$ in (7.10) and use again (7.16), (7.15) and (7.17) to get

where

$$
\psi_{t}(\bar{t}, \bar{x})+\eta G_{\eta}(\bar{x}, \tilde{y})-\bar{H}(\bar{x}, D \psi(\bar{t}, \bar{x})) \leq 0
$$

$$
G_{\eta}(\bar{x}, \tilde{y}):=\lim _{\varepsilon \rightarrow 0} G_{\varepsilon, \eta}(x, y)
$$


where and $G_{\varepsilon, \eta}$ is defined in (7.8).

Note that

$$
\begin{aligned}
G_{\eta}(\bar{x}, \tilde{y})=-b(\tilde{y}) \cdot D \chi(\tilde{y})- & \operatorname{tr}\left(\tau(\tilde{y}) \tau(\tilde{y})^{T} D^{2} \chi(\tilde{y})\right)-\eta\left|\tau(\tilde{y})^{T} D \chi(\tilde{y})\right|^{2} \\
& -2 \tau(\tilde{y})^{T} D w(\tilde{y}) \cdot D \chi(\tilde{y})-2 \tau(\tilde{y}) \sigma(\bar{x}, \tilde{y})^{T} D_{x} \psi(\bar{t}, \bar{x}) \cdot D \chi(\tilde{y}) .
\end{aligned}
$$

We observe that if $\tilde{y}$ is uniformly bounded in $\eta$, we send $\eta \rightarrow 0$ and we conclude

$$
\psi_{t}-\bar{H}(\bar{x}, D \psi(\bar{t}, \bar{x})) \leq 0 .
$$

Otherwise, if

$$
|\tilde{y}| \rightarrow+\infty \text { as } \eta \rightarrow 0,
$$

we prove analogously as in Lemma 7.3 (1) that for any $\eta$ small enough

$$
\lim _{\eta \rightarrow 0} G_{\eta}(\bar{x}, \tilde{y})=+\infty .
$$

Then we can suppose for $\eta$ small

$$
\eta G_{\eta}(\bar{x}, \tilde{y}) \geq 0
$$

and by coupling (7.20) with (7.18), we conclude again (7.19).

Finally we prove Lemma 7.3.

Proof of Lemma 7.3. First we prove Lemma 7.3 (1). Take $\eta, \varepsilon<1$ and consider $|y| \geq R_{1}$, where $R_{1}$ is defined in $(U)$. We analyse $G_{\varepsilon, \eta}$ term by term:

$$
-b(y) \cdot D_{y} \chi(y)-\eta\left|\tau(y)^{T} D_{y} \chi(y)\right|^{2} \geq 2 a|y|^{2}-2 a|b||y|-4 a^{2} T|y|^{2},
$$

by (7.6) and assumption $(U)$;

$$
-\operatorname{tr}\left(\tau(y) \tau(y)^{T} D^{2} \chi(y)\right)-2 \tau(y) \sigma(x, y)^{T} D_{x} \psi(t, x) \cdot D_{y} \chi(y) \geq-2 a K\left|D_{x} \psi(t, x)\right||y|-2 a K,
$$

where from now on we denote by $K>0$ a constant depending only on $\|\tau\|_{\infty},\|\sigma\|_{\infty}$ which may change from line to line. Note that $\left|D_{x} \psi(t, x)\right|$ is bounded uniformly in $\varepsilon$ by Lemma 7.2 (i) and the smoothness of $\psi$. We control the growth of the gradient of $w$ by the global estimate (5.2) proved in Proposition 5.2 and we get

$$
-2 \tau(y)^{T} D_{y} \chi(y) \cdot D_{y} w(y) \geq-4 a C K|y|,
$$

where $C$ is defined in (5.2). Then, by coupling all the previous estimates, we get

$$
G_{\varepsilon, \eta}(x, y) \geq\left(2 a-4 a^{2} T\right)|y|^{2}-2 a|b||y|-4 a C K|y|-2 a K\left|D_{x} \psi(t, x)\right||y|-2 a K .
$$

and by the second of (7.6), we complete the proof of Lemma 7.3 (1).

In order to prove Lemma 7.3 (2), we use again (5.2) of Proposition 5.2 to get

$$
\left.\left.\tau(y) \sigma(\bar{x}, y)^{T} D_{x} \psi(t, x)\right) \cdot D_{y} w(y) \geq-K C \mid D_{x} \psi(t, x)\right) \mid,
$$

where $C>0$ is defined in (5.2). Then, since $(t, x)$ are bounded in $\varepsilon$ by Lemma $7.2(\mathrm{i}), \tau, \sigma$ are bounded, and by the definition of $F_{\varepsilon}$ in $(7.11)$, we conclude

$$
F_{\varepsilon}(x, y) \geq-C^{\prime},
$$

for some constant $C^{\prime}>0$. The upper bound in Lemma 7.3 (2) is deduced by similar arguments. 


\section{ApPlichtions TO OPTION PRICING AND IMPLIED VOLATILITY}

In this section we derive a large deviation principle for the process $X_{t}^{\varepsilon}$ defined in (2.3). As applications, we give estimates of option prices near maturity and an asymptotic formula for the implied volatility. Similar financial applications were also given in [7], in particular we refer to Theorem 7.1, Corollaries 8.1 and 8.2. However, at least to our point of view, these financial applications take on particular relevance in the present paper due to the unbounded setting, which provides a more realistic model for volatility as appearing in financial markets. We refer to the introduction of the present paper for further discussions.

Throughout this section, we assume that there exists $\nu>0$ such that

$$
\left|\sigma(x, y)^{T} \xi\right|^{2} \geq \nu|\xi|^{2}, \quad \text { for all } x \in \mathbb{R}^{n}, y \in \mathbb{R}^{m}, \xi \in \mathbb{R}^{n} .
$$

Remark 8.1. With respect to assumption (S1), where the uniform non degeneracy of $\sigma$ is supposed only the fast variable and for $\alpha>2,(8.1)$ assumes the uniform non degeneracy in both variable and for all ranges of $\alpha$. Assumption 8.1 is supposed only in this section in order to ensure the coercivity of the effective Hamiltonian and the well-definition of the rate function, see $(8.2)$.

Before giving our results, let us recall the definition of a large deviation principle. Given a family of probability measures $\left\{\mu_{\varepsilon}\right\}$, a large deviation principle characterizes the limiting behaviour as $\varepsilon \rightarrow 0$ of $\left\{\mu_{\varepsilon}\right\}$ in terms of a rate function through asymptotic upper and lower exponential bounds on the values that $\mu_{\varepsilon}$ assigns to measurable subsets of $\mathbb{R}^{n}$. The precise definition is recalled in the following.

Definition 8.1. Let $\mathcal{B}$ denotes the Borel $\sigma$-field of $\mathbb{R}^{n}$.

(i) A rate function $I$ is a lower semicontinuous map $I: \mathbb{R}^{n} \rightarrow[0, \infty]$ and it is a good rate function if for all $\alpha \in[0, \infty)$, the level set $\Psi_{I}(\alpha):=\{x: I(x) \leq \alpha\}$ is compact.

(ii) A family of probability measures $\left\{\mu_{\varepsilon}\right\}$ satisfies the large deviation principle with a rate function $I$ if, for all $B \in \mathcal{B}$,

$$
-\inf _{x \in B^{\circ}} I(x) \leq \liminf _{\varepsilon \rightarrow 0} \varepsilon \log \mu_{\varepsilon}(B) \leq \limsup _{\varepsilon \rightarrow 0} \varepsilon \log \mu_{\varepsilon}(B) \leq-\inf _{x \in \bar{B}} I(x) .
$$

For each $x_{0} \in \mathbb{R}^{n}$ and $t>0$, we define

$$
I\left(x ; x_{0}, t\right):=\inf \left[\int_{0}^{t} \bar{L}(\xi(s), \dot{\xi}(s)) d s \mid \xi \in A C(0, t), \xi(0)=x_{0}, \xi(t)=x\right]
$$

where $\bar{L}$ is the effective Lagrangian, i.e. for $x \in \mathbb{R}^{n}, \bar{L}(x, q)=\max _{p \in \mathbb{R}^{n}}\{p \cdot q-\bar{H}(x, p)\}$.

Remark 8.2. Note that under (8.1) and by (3.19), the effective Hamiltonian $\bar{H}$ is coercive.

Remark 8.3. The rate function I satisfies the following properties:

(a) I is continuous in the variable $x$ (see, e.g. [7], [22]) and is a nonnegative function such that $I\left(x_{0} ; x_{0}, t\right)=0$.

(b) I satisfies the following growth condition for some $C>0$ and all $x, x_{0} \in \mathbb{R}^{n}$

$$
\frac{1}{4 C} \frac{\left|x-x_{0}\right|^{2}}{t} \leq I\left(x ; x_{0}, t\right) \leq \frac{1}{4 \nu} \frac{\left|x-x_{0}\right|^{2}}{t},
$$

where $\nu$ is defined in (S1). The proof of (8.3) easily follows by (3.19) and by the definition of I (8.2) (see also [7], Remark 7 for more details).

(c) If $\sigma$ does not depend on $x$, i.e. $\bar{H}=\bar{H}(p)$, the rate function in (8.2) is

$$
I\left(x ; x_{0}, t\right)=t \bar{L}\left(\frac{x-x_{0}}{t}\right) \text {. }
$$


(d) If $\sigma$ does not depend on $x$ and $n=1, \mathrm{I}$ is a monotone non decreasing function of $x$ when $x>x_{0}$. Analogously, $\mathrm{I}$ is a monotone non increasing function of $x$ when $x<x_{0}$.

The firs result is the following large deviation principle for the process $X_{t}^{\varepsilon}$ in $(2.3)$.

Theorem 8.1. Let assumption (U), (S1) and (S2) hold. Let $\left(X^{\varepsilon}, Y^{\varepsilon}\right)$ be the process defined in (2.3) with initial position $X_{0}^{\varepsilon}=x_{0}$ and $Y_{0}^{\varepsilon}=y_{0}$. Then for every $t>0$, a large deviation principle holds for $\left\{X_{t}^{\varepsilon}: \varepsilon>0\right\}$ with speed $\frac{1}{\varepsilon}$ and good rate function $I\left(x ; x_{0}, t\right)$. In particular, for any open set $B \subseteq \mathbb{R}^{n}$

$$
\lim _{\epsilon \rightarrow 0} \epsilon \log P\left(X_{t}^{\varepsilon} \in B\right)=-\inf _{x \in B} I\left(x ; x_{0}, t\right) .
$$

Proof. The proof follows by the same arguments to Theorem 7.1 [7], namely by proving the exponential tightness of $\left\{X_{t}^{\varepsilon}\right\}$ and then applying Bryck inverse Varadhan lemma with $\left\{\mu_{\varepsilon}\right\}$ given by the laws of $\left\{X_{\varepsilon}\right\}$ and $\Lambda_{h}$ given by $v_{\varepsilon}$. A crucial step is the convergence of $v_{\varepsilon}$, which is proved in Theorem 7.1. We omit the details since the proof of the exponential tightness of $\left\{X_{t}^{\varepsilon}\right\}$ is the same as in [7].

In the following we give estimates of option prices near maturity (Corollary 8.2) and an asymptotic formula for the implied volatility (Corollary 8.3). The proofs are omitted since they follow by direct applications of Theorem 8.1 (and moreover, similar arguments were used in [7] Corollaries 8.1 and 8.2). We refer also to [28] for a detailed review of the results of the present paper and of [7]. First let us set some notation in order to introduce the results. Let $S_{t}^{\varepsilon}$ be the asset price, evolving according to the following stochastic differential system

$$
\begin{cases}d S_{t}^{\varepsilon}=\varepsilon \xi\left(S_{t}^{\varepsilon}, Y_{t}^{\varepsilon}\right) S_{t}^{\varepsilon} d t+\sqrt{2 \varepsilon} \zeta\left(S_{t}^{\varepsilon}, Y_{t}^{\varepsilon}\right) S_{t}^{\varepsilon} d W_{t} & S_{0}^{\varepsilon}=S_{0} \in \mathbb{R}_{+} \\ d Y_{t}^{\varepsilon}=\varepsilon^{1-\alpha} b\left(Y_{t}^{\varepsilon}\right) d t+\sqrt{2 \varepsilon^{1-\alpha}} \tau\left(Y_{t}^{\varepsilon}\right) d W_{t} & Y_{0}^{\varepsilon}=y_{0} \in \mathbb{R}^{m}\end{cases}
$$

where $\alpha>2, \tau, b$ are as in (2.3) and $\xi: \mathbb{R}_{+} \times \mathbb{R}^{m} \rightarrow \mathbb{R}, \zeta: \mathbb{R}_{+} \times \mathbb{R}^{m} \rightarrow \mathbf{M}^{1, r}$ are Lipschitz continuous bounded functions, periodic in $y$. Observe that $S_{t}^{\varepsilon}>0$ almost surely if $S_{0}>0$. We define $X_{t}^{\varepsilon}=\log S_{t}^{\varepsilon}$. Then $\left(X_{t}^{\varepsilon}, Y_{t}^{\varepsilon}\right)$ satisfies (2.3) with

$$
\phi(x, y)=\xi\left(e^{x}, y\right)-\zeta\left(e^{x}, y\right) \zeta^{T}\left(e^{x}, y\right) \quad \sigma(x, y)=\zeta\left(e^{x}, y\right)
$$

We consider out-of-the-money call option by taking

$$
S_{0}<K \quad \text { or } \quad x_{0}<\log K .
$$

Similarly, by considering out-of-the-money put options, one can obtain the same formula for $S_{0}>K$.

Corollary 8.2. Let assumptions (U), (S1) and (S2) hold. Suppose that $S_{0}<K$. Then, for fixed $t>0$

$$
\lim _{\varepsilon \rightarrow 0^{+}} \varepsilon \log E\left[\left(S_{t}^{\varepsilon}-K\right)^{+}\right]=-\inf _{y>\log K} I\left(y ; x_{0}, t\right) .
$$

We recall that given an observed European call option price for a contract with strike price $K$ and expiration date $T$, the implied volatility $\sigma_{\varepsilon}\left(t, \log K, x_{0}\right)$ is defined to be the value of the volatility parameter that must go into the Black-Scholes formula to match the observed price. We have the following corollary.

Corollary 8.3. Let assumptions (U), (S1) and (S2) hold. Then

$$
\lim _{\varepsilon \rightarrow 0^{+}} \sigma_{\varepsilon}^{2}\left(t, \log K, x_{0}\right)=\frac{\left(\log K-x_{0}\right)^{2}}{2 \inf _{y>\log K} I\left(y ; x_{0}, t\right) t} .
$$


Remark 8.4. Note that the infimum in the right-hand side of (8.7), is always positive by assumption (8.6) and by (8.3).

\section{ACKNOWLEDGEMENT}

Part of this work was developed while the author was a Ph.D student at the Dept. of Mathematics of the Univ. of Padua. The author wants to express her deep gratitude to M. Bardi and A. Cesaroni for suggesting the problem and for the important help given. The author wishes also to warmly thank Guy Barles and Olivier Ley for the interesting suggestions which strongly contributed to the improvement of some proofs.

\section{REFERENCES}

[1] O. Alvarez, M. Bardi: Viscosity solutions methods for singular perturbations in deterministic and stochastic control, SIAM J. Control Optim. 40 (2001/02), 1159-1188.

[2] O. Alvarez, M. Bardi: Singular perturbations of nonlinear degenerate parabolic PDEs: a general convergence result, Arch. Ration. Mech. Anal. 170 (2003), 17-61.

[3] O. Alvarez, M. Bardi: Ergodicity, stabilization, and singular perturbations for Bellman-Isaacs equations, Mem. Amer. Math. Soc. (2010), no. 960, vi+77 pp.

[4] O. Alvarez, M. Bardi, C. Marchi, Multiscale problems and homogenization for second-order HamiltonJacobi equations, J. Differential Equations 243 (2007), 349-387.

[5] M. Arisawa, P.-L. Lions, On ergodic stochastic control, Comm. Partial Differential Equations 23 (1998), 2187-2217.

[6] M. Bardi, A. Cesaroni, L. Manca,Convergence by viscosity methods in multiscale financial models with stochastic volatility, Siam J. Financial Math. 1 (2010), 230-265.

[7] M. Bardi, A. Cesaroni, D. Ghilli, Large deviations for some fast stochastic volatility models by viscosity methods, Discrete Contin. Dyn. Syst. A. 35 (2015), n. 9, 3965-3988.

[8] G. Barles, A weak bernstein method for fully nonlinear elliptic equations, Differential Integral equations 4, (1991), no. 2, 241-262.

[9] G. Barles, $C^{0, \alpha}$-regularity and estimates for solutions of elliptic and parabolic equations by the IshiiLions method, Gakuto International Series, Mathematical Sciences and Applications, 30 (2008), 33-47.

[10] G. Barles, A short proof of the $C^{0, \alpha}$-regularity of viscosity subsolutions for superquadratic viscous Hamilton-Jacobi equations and applications, Nonlinear Anal., 73 (2010), no.1, 31-47.

[11] G. Barles, Solutions de viscosité des équations de Hamilton-Jacobi, Mathématiques and Applications 17, Springer-Verlag.

[12] G. Barles, H. Ishii, and H. Mitake, A new PDE approach to the large time asymptotics of solutions of Hamilton-Jacobi equations, Bull. Math. Sci. 3 (2013), no. 3, 363-388.

[13] G. Barles, B. Perthame, Comparison principle for Dirichlet-type Hamilton-Jacobi equations and singular perturbations of degenerated elliptic equations. Appl. Math. Optim. 21 (1990), 21-44.

[14] G. Barles, P. Souganidis, Space-time periodic solutions and long-time behavior of solutions of quasilinear parabolic equations, SIAM J. Math. Anal. (electronic) 32 (2001), no.6, 1311-1323.

[15] A. Bensoussan, Perturbation Methods in Optimal Control, John Wiley and Sons, Montrouge, France, 1988.

[16] V. S. Borkar and V. Gaitsgory, Singular perturbations in ergodic control of diffusions, SIAM J. Control Optim., 46 (2007), no.5, 15621577.

[17] I. Capuzzo Dolcetta, F. Leoni, and A. Porretta, Hölder estimates for degenerate elliptic equations with coercive Hamiltonians, Trans. Amer. Math. Soc. 362 (2010), no.9, 4511-4536.

[18] P. Cardaliaguet, A note on the regularity of solutions of Hamilton-Jacobi equations with superlinear growth in the gradient variable ESAIM Control Optim. Calc. Var. 15 (2009), no.2m 367-376.

[19] P. Cardaliaguet and L. Silvestre, Hölder continuity to Hamilton-Jacobi equations with superquadratic growth in the gradient and unbounded right-hand side, Comm. Partial Differential Equations 37 (2012), 1668- 1688.

[20] M.G. Crandall, H. Ishii, P.-L. Lions, User's guide to viscosity solutions of second order partial differential equations, Bull. Amer. Math. Soc. (N.S.) 27 (1992), 1-67.

[21] F. Da Lio, O. Ley, Uniqueness results for second order Bellman-Isaacs equations under quadratic growth assumptions and applications, SIAM J. Control Optim. 45 (2006), no 1, 74-106.

[22] G. Dal Maso, H. Frankowska, Value functions for Boltza problems with discontinuous lagrangian and Hamilton-Jacobi inequality, ESAIM Control Optim. Calc. Var. 5 (2000), 369-393. 
[23] L. C. Evans, The perturbed test function method for viscosity solutions of nonlinear PDE, Proc. Roy. Soc. Edinburgh Sect. A 111 (1989), 359-375.

[24] L. C. Evans, H. Ishii, A PDE approach to some asymptotic problems concerning random differential equations with small noise intensities. Ann. Inst. H. Poincaré Anal. Non Linéaire 2 (1985), 1-20.

[25] J. Feng, J.-P. Fouque, R. Kumar, Small time asymptotic for fast mean-reverting stochstic volatility models, Ann. Appl. Probab. 22 (2012), no. 4, 1541-1575.

[26] J. Feng, T. G. Kurtz, Large deviations for stochastic processes. American Mathematical Society, Providence, RI, 2006.

[27] J.-P. Fouque, G. Papanicolaou, K.R. Sircar, Derivatives in financial markets with stochastic volatility. Cambridge university press, Cambridge, 2000.

[28] D. Ghilli, Some results in nonlinear PDEs: large deviations problems, nonlocal operators and stability for some isoperimetric problems, Ph.D thesis (2016), Dept. of Mathematics, Univ. of Padua.

[29] R. Z. Hasminskii, Stochastic Stability of Differential Equations, Sijthoff and Noordhoff, Alphen aan denRijn, The Netherlands, Germantown, MD (1980).

[30] N. Ichihara, Recurrence and transience of optimal feedback processes associated with Bellman equations of ergodic type, SIAM J. Control Optim. 49 (2011), no. 5, 19381960.

[31] N. Ichihara, S. Sheu, Large time behavior of solutions of Hamilton-Jacobi-Bellman equations with quadratic nonlinearity in gradient, Siam J. Math. Anal. 45 (2013), no.1, 279306.

[32] H. Ishii, P.-L. Lions, Viscosity solutions of fully nonlinear second-order elliptic partial differential equations, J. Differential Equations 83 (1990), no.1, 26-78.

[33] H. Kaise, S. Sheu, On the structure of solution of ergodic type Bellman equation related to risksensitive control, Ann. Probab. 34 (2006), no. 1, 284-320.

[34] O. Ley, V. Duc Nguyen, Gradient bounds for nonlinear degenerate parabolic equations and application to large time behavior of systems, Nonlinear Analysis: Theory, Methods and Applications, Elsevier 130 (2016), 76-101.

[35] P.-L. Lions, Generalized solutions of Hamilton-Jacobi equations, Pitman (Advanced Publishing Program), Boston, Mass.(1982).

[36] P.-L. Lions, M. Musiela, Ergodicity of Diffusion Processes, manuscript (2002).

[37] P. -L. Lions, P. E. Souganidis, Homogenization of degenerate second-order PDE in periodic and almost periodic environments and applications, Ann. I. H. Poincar-AN 22 (2005), 667-677.

[38] L. Lorenzi, M. Bertoldi, Analytical Methods for Markov Semigroups, Pure and Applied Mathematics (Boca Raton), 283. Chapman and Hall/CRC, Boca Raton, FL, 2007. xxxii+526 pp.

[39] E. Pardoux, A. Yu. Veretennikov, On the Poisson equation and diffusion approximation, I, Ann. Probab. 29 (2001), no.3, 10611085. Ann. Probab. 31 (2003), no.3, 11661192

[40] E. Pardoux, A. Yu, Veretennikov, On the Poisson equation and diffusion approximation, II, Ann. Probab. 31 (2003), no.3, 11661192.

[41] E. Pardoux, A. Yu, Veretennikov, On the Poisson equation and diffusion approximation, III, Ann. Probab. 33 (2005), no.3, 11111133.

[42] M. V. Safonov, On the classical solution of nonlinear elliptic equations of second order, Izv. Akad. Nauk SSSR Ser. Mat. 52 (1988), no. 6, 12721287.

[43] N.S. Trudinger, On regularity and existence of viscoity solutions of nonlinear second order, elliptic equations, Partial differential equations and the Calculus of variations: Essays in Honor of Ennio de Giorgi, Progress in Nonlinear Differential Equations and their Applications, Birkhaüser Boston Inc., (1989).

[44] A. Yu. Veretennikov, On large deviations for SDEs with small diffusion and averaging. Stochastic Process. Appl. 89 (2000), no. 1, 69-79.

Daria Ghilli, Karl-Franzens University of Graz, Universitatplatz 3, Austria E-mail address: daria.ghilli@uni-graz.at 